\title{
A Cell Outage Management Framework for Dense Heterogeneous Networks
}

\author{
Oluwakayode Onireti, Member, IEEE, Ahmed Zoha, Member, IEEE, Jessica Moysen, Member, IEEE, \\ Ali Imran, Member, IEEE, Lorenza Giupponi, Senior Member, IEEE, Muhammad Ali \\ Imran, Senior Member, IEEE and Adnan Abu-Dayya, Senior Member, IEEE
}

\begin{abstract}
In this paper, we present a novel cell outage management (COM) framework for heterogeneous networks (HetNets) with split control and data planes -a candidate architecture for meeting future capacity, quality of service and energy efficiency demands. In such architecture, the control and data functionalities are not necessarily handled by the same node. The control base stations (BSs) manage the transmission of control information and user equipment (UE) mobility, while the data BSs handle UE data. An implication of this split architecture is that, an outage to a BS in one plane has to be compensated by other BSs in the same plane. Our COM framework addresses this challenge by incorporating two distinct cell outage detection (COD) algorithms to cope with the idiosyncrasies of both the data and control planes. The COD algorithm for control cells leverages the relatively larger number of UEs in the control cell to gather large scale minimize drive testing (MDT) reports data, and detects outage by applying machine learning and anomaly detection techniques. To improve outage detection accuracy, we also investigate and compare the performance of two anomaly detecting algorithms, i.e. $k$ - nearest neighbor and local outlier factor based anomaly detector, within the control COD. On the other hand, for data cells COD, we propose a heuristic grey-prediction based approach, which can work with the small number of UEs in the data cell, by exploiting the fact that the control BS manages UE-data BS connectivity, by receiving a periodic update of the received signal reference power (RSRP) statistic between the UEs and data BSs in its coverage. The detection accuracy of the heuristic data COD algorithm is further improved by exploiting the Fourier series of residual error that is inherent to grey prediction model. Our COM framework integrates these two COD algorithms with a cell outage compensation (COC) algorithm which can be applied to both planes. Our COC solution utilizes an actor critic (AC) based reinforcement learning (RL) algorithm, which optimizes the capacity and coverage of the identified outage zone in a plane, by adjusting the antenna gain and transmission power of the surrounding BSs in that plane. The simulation results show that the proposed framework can detect both data and control cell outage, and also compensate for the detected outage in a reliable manner.
\end{abstract}

Index Terms-Self-organizing network, self-healing, cell outage management, cell outage detection, cell outage compensation, heterogeneous cellular network.

Copyright (c) 2015 IEEE. Personal use of this material is permitted. However, permission to use this material for any other purposes must be obtained from the IEEE by sending a request to pubs-permissions@ieee.org.

O. Onireti and M. A. Imran are with the Institute for Communication Systems, University of Surrey, UK (e-mail: \{o.s.onireti, m.imran\}@ surrey.ac.uk).

A. Zoha and A. Abu-Dayya are with Qatar Mobility Innovations Center, Qatar (e-mail: \{ahmedz,adnan\}@qmic.com).

J. Moysen and L. Giupponi are with the Centre Tecnòlogic Telecomunicacions Catalunya, Spain (e-mail:\{lorenza.giupponi, jessica.moysen\}@cttc.es).

A. Imran is with University of Oklahoma, USA (e-mail: ali.imran@ou.edu).

\section{INTRODUCTION}

$\mathbf{R}$ ECENTLY, extensive research and standardization efforts have been channeled towards the definition of the paradigm of self-organizing network (SON), which aim at achieving a substantial reduction in capital and operational expenditures (CAPEX \& OPEX) by reducing human involvement in network operational tasks, while optimizing the network coverage, capacity and quality of service [1], [2]. SONs aim to replace the manual operational processes that have been executed in legacy cellular networks since their conception, such as configuration, post-deployment optimization and troubleshooting, with autonomous functions called SON functions, such as self-configuration, self-optimization and self-healing [1]-[3]. The main task within self-healing functionality is autonomous cell outage detection and its compensation. Traditionally cell outages have been detected manually. In some cases, cell outage can be detected by the manual analysis of fault alarms at operations and maintenance center (OMC), while other detections require site visits or drive testing. This is an expensive process. In addition, it may take hours or days for the cell outage to be detected, thus resulting in pronounced reduction in capacity and quality of service, and coverage gap [4], [5]. Once detected, the outage is compensated in ad-hoc and manual fashions, making the whole process extremely inefficient and unreliable. With increasing scale of networks, automatic detection and compensation of cell outage has become a necessity, and, it has been included in recent 3GPP releases [6]. Therefore, the SON paradigm aims to replace these manual tasks with an autonomous process referred to cell outage management (COM) [7]-[12]. COM can be further subdivided into cell outage detection (COD) and cell outage compensation (COC). COD aims to autonomously detect outage cells, i.e. cells that are not operating properly due to possible failures, e.g. external failure such as power supply or network connectivity, or even misconfiguration [4][6], [13]. On the other hand, COC refers to the automatic mitigation of the degradation effect of the outage by appropriately adjusting suitable radio parameters, such as the pilot power, antenna tilt and azimuth of the surrounding cells. The degree of compensation is usually dictated by the operators policies, which also specify the level of performance that must be satisfied in the outage region [9].

A few algorithms have already been proposed in literature, e.g in [4], [13]-[18] and [8]-[11], for COD and COC, respectively. The COD problem has been addressed in [4] by 
leveraging the neighbor cell list (NCL) reports to construct a visibility graph, whose topology changes are used to detect cells that are experiencing outage. In [13] a weighted combination of three hypothesis, which was based on the distribution of channel quality indicator (CQI), time correlation of CQI differential and registration request frequency, was used in detecting cell outage. Just recently interest has emerged in applying methods from the machine learning domain such as clustering algorithms [16] as well as Bayesian networks [18] to automate the detection of faulty cell behavior. Coluccia et al. [17] analyzed the variations in the traffic profiles for $3 \mathrm{G}$ cellular systems to detect real world traffic anomalies. In terms of COC, the authors in [8], [9] investigated the effectiveness of control parameters such as the reference signal power, antenna tilt, scheduling parameters and the uplink target received power level in mitigating the effect of cell outage. In [10], the authors proposed an autonomic particle swarm compensation algorithm with fast convergence speed, which is a key requirement in COC.

All these works have focused on the traditional homogeneous deployments, where only macro cells are deployed. However, future cellular deployments are expected to be heterogeneous and extremely dense. In this context, macro cells will provide the user equipment (UE) with ubiquitous experience, while dense small cell deployments operating in bandwidths with heterogeneous characteristics will facilitate high data rate transmissions to a reduced number of UEs. At the same time, conventional heterogeneous deployments pose a number of challenges in terms of network management and energy consumption, as a result of the increased number of cells [19]. In order to mitigate these challenges, a new heterogenous network (HetNet) architecture with split control and data planes has been recently proposed as a candidate architecture for 5G [20]-[27]. In such architecture, the control and data planes are separated and are not necessarily handled by the same node. Consequently, this gives the network operator more flexibility, since the small/data cells can be activated on demand to deliver UE-specific data only when and where needed, while the macro/control cells manage UEs connectivity and mobility [24]. Thus, the separated plane architecture allows for improved mobility management performance, since the radio resource control (RRC) layers of the UEs and other control messaging, such as paging, will be handled by the control cells. In addition, the energy consumption is improved, since the proposed architecture also leads to longer data cell sleep periods, due to their on demand activation. Note that contrarily to the newly proposed HetNet architecture, the RRC layers of all UEs in the conventional HetNet are handled by their serving cell, which could be either a small or macro cell.

Only recently, [5] proposed a cooperative COD scheme for small cells in a conventional HetNet, while [28] proposed a COD scheme for detecting both macro cell and small cell outages in a conventional HetNet. However, accurate small cell outage detection with [28] requires fairly large number of UEs in each small cell, which therefore limits its usage. Furthermore, COC solutions for HetNets have been proposed in [29], [30]. In [29], handover to macro is proposed as a solution for an outage caused by a small cell, while a collaborative resource allocation approach is proposed for UEs that cannot be served by the macro cell in [30]. Though individual COC and COD algorithms have been presented in literature for conventional deployments, a complete COM framework, particularly for HetNet with split control and data plane, is still missing. The main difference in the COM framework in HetNets with split control and data planes and the conventional HetNet is in their architecture. In the traditional HetNet, control and data functionalities are provided to the UE by the same node, whereas these functionalities are provided by separate nodes in the split architecture. Hence, in the conventional architecture outage to a node can be compensated by any other node, whereas in the split architecture an outage to a node can only be compensated by another node that provides the same functionalities. In this paper, we propose a complete COM framework, composed of concrete solutions for COD and COC, for a HetNet with separated control and data plane functionalities, and with different spectrum resources allocated to each plane.

Our framework has two distinct COD algorithms to cope with the peculiarities of data and control cells. Since control cells tend to have a large number of UEs, we exploit large scale collection of minimization of drive test (MDT) reports, introduced by 3GPP in [31], and we apply machine learning based anomaly detection schemes for control COD. Control COD thus can be implemented at the OMC level. In control COD solution, we compare the performance of two anomaly detecting algorithms, i.e., $k$-nearest neighbor anomaly detector ( $k$-NNAD) and local outlier factor anomaly detector (LOFAD), to find the best. However, the same COD scheme cannot be applied for data cells, as the number of users will not be large enough to constitute reliable training models for underlying anomaly detection techniques. Hence, we propose a heuristic data COD scheme, which works despite of small number of UEs in the data cell, by exploiting a grey-prediction model (GM) for detecting data cell outage.

Once the outage is properly detected, we need to implement an online automatic $\mathrm{COC}$ scheme to continue serving the UEs in the outage area. Considering the acute dynamics of the always varying wireless environment in general, and the high variability in terms of load fluctuations, in dense wireless deployments, we propose an actor critic (AC) based reinforcement learning (RL) algorithm, which allows to learn online, through interactions with the surrounding environment, the best possible policy to compensate the outage. The solution is based on optimizing the coverage and capacity of the identified outage zone, by adjusting the gains of the antennas through electrical tilt, and downlink transmission power of the surrounding BSs in that plane. The proposed COC algorithm can be applied independently in each plane, as different spectrum resources are allocated to each plane.

The main contribution of this paper can be summarized as follows: 1) We propose a novel COM framework for the HetNet with split control and data plane, 2) We design, evaluate and compare novel COD solutions for detecting control and data cell outages in the split architecture and 3) We define an AC-RL algorithm, which can be used for both the control and data COC in the split architecture. The remainder 
of this paper is organized as follows. In Section II, we present the system architecture, which includes description of the split architecture, system model and COM framework. In Section III, we propose a low dimensional embedding of MDT measurement for control COD. In Section IV, we introduce a heuristic based data COD scheme. Section V presents an AC based RL algorithm for both control and data COC. In Section VI, we present extensive simulations to substantiate the performance of our proposed COM framework for HetNets with separated control and data planes. Finally, Section VII concludes this paper.

\section{System ARChitecture}

\section{A. Split Architecture}

We consider the new paradigm of HetNets architecture where the data and control/signaling planes are decoupled at the air interface, as recently proposed in [20]-[24]. The control plane provides ubiquitous network access and is made up of macro base station (BS)s, which we refer to as control BSs. On the other hand, data plane supports high data rate transmission and is composed of the small BSs, which we call data BSs. The control plane handles UE connectivity as well as different radio-specific functions, which primarily cover: 1) RRC connection management, 2) system information broadcast and synchronization, 3) configuration and measurement reporting, and 4) cell handover and network controlled mobility. In contrast, the data plane handles UE specific data, and its functionalities are unicast and synchronization [23], [24]. Consequently, UEs requiring high data rate transmission are connected to both the control and data BSs, while low rate UEs are connected to just the control BS.

\section{B. System Model}

We consider that the control and data BSs are operated on separate frequency carriers, so that there is no interference between the two planes. We assume that the HetNet is composed of a set of $\mathcal{M}$ macro BSs/control BSs and $\mathcal{F}$ small BSs/data BSs, where $M=|\mathcal{M}|$ control BSs form a regular hexagonal network layout with inter-site distance $D$ and provides coverage over the entire network. We denote the transmission power vector of control BS $m \in \mathcal{M}$ by $\mathbf{p}^{m}=\left(p_{1}^{m}, \ldots, p_{R}^{m}\right)$, where $p_{r}^{m}$ is the downlink transmission power of resource blocks (RB) $r$ and the maximum transmission power of each control $\mathrm{BS}, P_{\max }^{\mathrm{m}}$, is such that $\sum_{r=1}^{R} p_{r}^{m} \leq P_{\max }^{\mathrm{m}}$. The $F=|\mathcal{F}|$ data BSs are randomly distributed. Similarly, we denote the transmission power vector of data BS $f \in \mathcal{F}$ by $\mathbf{p}^{f}=\left(p_{1}^{f}, \ldots, p_{R}^{f}\right)$, where $p_{r}^{f}$ is the downlink transmission power of RB $r$ and the maximum transmission power of each data $\mathrm{BS}, P_{\max }^{f}$, is such that $\sum_{r=1}^{R} p_{r}^{f} \leq P_{\max }^{\mathrm{f}}$. We also consider that $U_{m}$ and $U_{f}$ UEs are provided with service by the $\mathcal{M}$ control BSs and $\mathcal{F}$ data $\mathrm{BSs}$, respectively. The multi user resource assignment to the $R \mathrm{RBs}$ in a plane is carried out by a fractional frequency reuse (FFR) scheduler and each of the UEs in the plane is assigned a CQI value. The FFR scheme, at the beginning of each subframe divides each cell into two regions, and assigns a certain group of RBs to the inner region and another, orthogonal, to the outer region, at the border of the cell. The inner regions of all the cells in the scenario are covered by the same spectrum $(f)$, while the outer regions are assigned orthogonal RBs such as $\left\{f_{1}, \ldots, f_{7}\right\}$, as it is shown in Fig. 1 (FFR1). The latter also represents the amount of bandwidth reserved for the outage zone during COC (FFR2 in Fig. 1).

\section{COM Framework}

In the HetNet architecture described earlier in Section II-B, outage can occur to a BS in either the control or data plane, i.e, control or data BS outage, thus leading to a degradation in system performance. In order to alleviate the effect of such outage, the BS in outage has to first be detected. This can be achieved by monitoring deviations from the key performance indicator (KPI) measurement report of the fault free network. Thereafter, the parameters of BSs neighboring the outage BS in a given plane, can be adjusted according to the operators policy so as to compensate for the outage situation. Hence, we propose a COM framework, which primarily consists of the COD and COC stages.

1) $C O D$ : As mentioned earlier, active high data rate UEs are served by both the data and control BS, while the low rate UEs are served by only the control BS. This implies that all UEs maintain connectivity with the control BS. Furthermore, as a result of the split of the control and data planes, the control and data cell outages are independent of each other, hence, the detection of a cell outage in each plane is executed independently of the other. As shown in Fig. 1, our framework has two distinct COD algorithms to cope with the idiosyncrasies of the control and data cell. The control cell tend to have large number of users, so a large scale data collection and machine learning is used for the control COD. Consequently, our control COD scheme is based on the MDT functionality, where all UEs report their MDT measurements, which include reference signal received power (RSRP) of the serving and neighboring cells, to the OMC vis respective BS. A normal network profile is built based on the measurements in a non-outage scenario. The control COD is then performed at the OMC, by using anomaly detection algorithms, such as $k$-NNAD and LOFAD, on the actual network profile.

However, this approach is not applicable for data COD, where UE statistics are sparse, due to the small number of UEs connected to each data BS. The control BS knows the location of every data BS in its coverage and it can passively monitor the RSRP measurements of every UE-data BS association within its coverage. Consequently, a heuristic scheme, which can effectively leverage on the small number of reports and the fact that the control BS can monitor the UE-data BS association, is used for the data COD. The data COD is executed at the control BS and is triggered when the control BS detects irregularities in UE-data BS associations, while the actual detection is performed by using a GM algorithm.

2) $C O C$ : After the control or data cell outage has been detected by the OMC or by the control BS, respectively, an AC-RL COC scheme is implemented independently in both planes, as shown in Fig. 1. Note that no inter-plane interference is experienced since both planes are operated on separate carrier frequencies and, hence, the COC implementation in one plane has no impact on the other plane. 


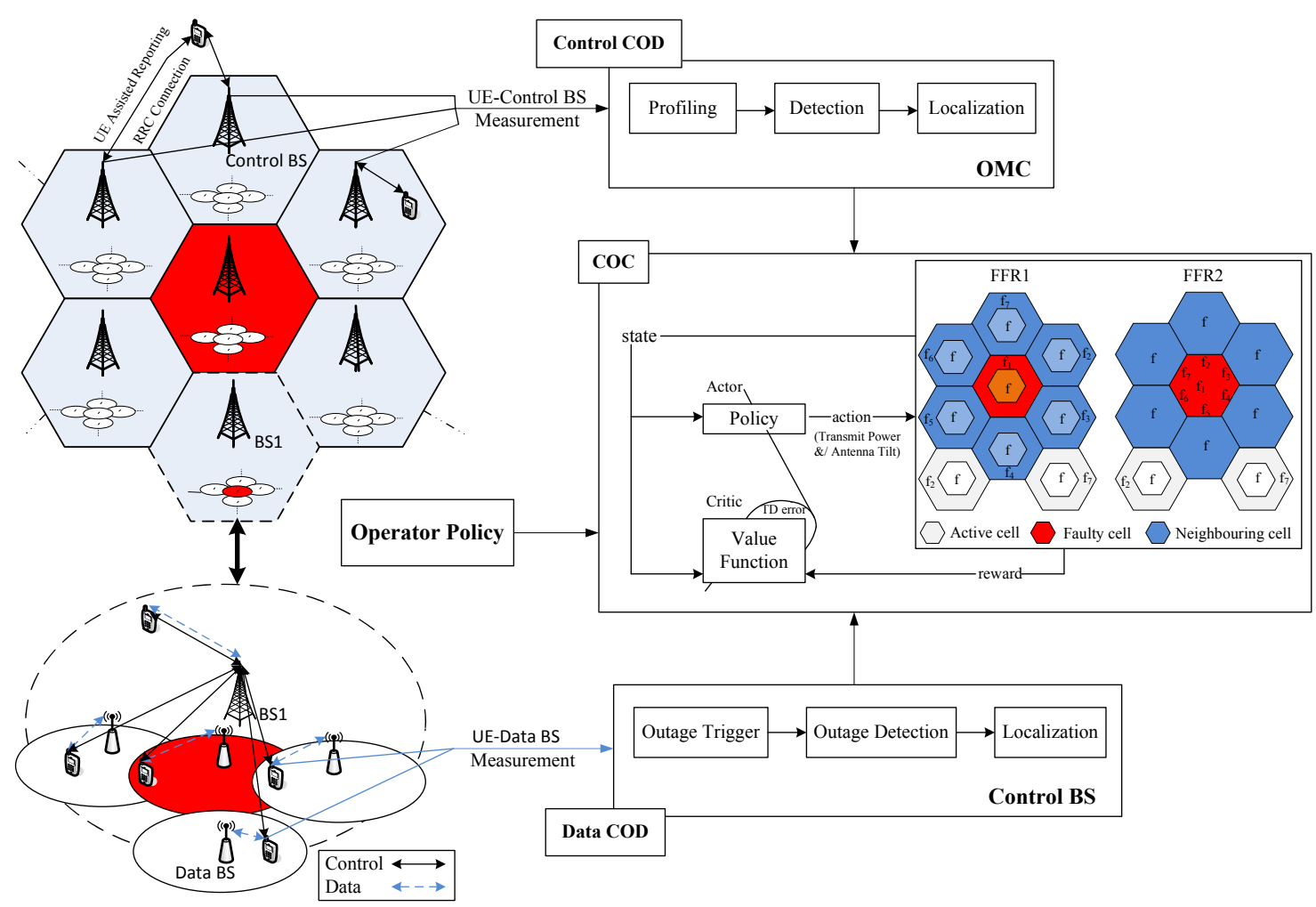

Fig. 1: An overview of COM framework

\section{CONTROL COD VIA LOW-DIMENSIONAL EMBEDDING OF MDT MEASUREMENT}

The main idea behind our control COD framework is to use the MDT reports acquired from a fault-free operating scenario to profile the behavior of the network. The goal is to use the learned profile to address the control cell outage autonomously. The proposed control COD framework adopts a four step based approach: 1) measurement, 2) profiling, 3) detection and 4) localization. Each step is further elaborated in the following subsections.

\section{A. Measurement}

The MDT reporting schemes have been defined in LTE Release 10 [31], [32]. The release proposes to construct a data base of MDT reports from the network using Immediate or Logged MDT reporting configuration. In this study, the UEs are configured to report the cell identification and radiomeasurement data to the target control BS, based on immediate MDT configuration procedure as shown in Fig. 2. The signaling flow of the MDT reporting procedure consists of: 1) configuration, 2) measurement, 3) reporting and 4) storing phase. The UE is first configured to perform measurements periodically, as well as whenever an A2 event (i.e., serving cell becomes worst than a threshold) occurs. Subsequently, the UE performs measurements as specified in Table I and further report them to the control BS. The control BS, after retrieving these measurements, further appends time and wide-band CQI to these measurements and forward them to trace collection

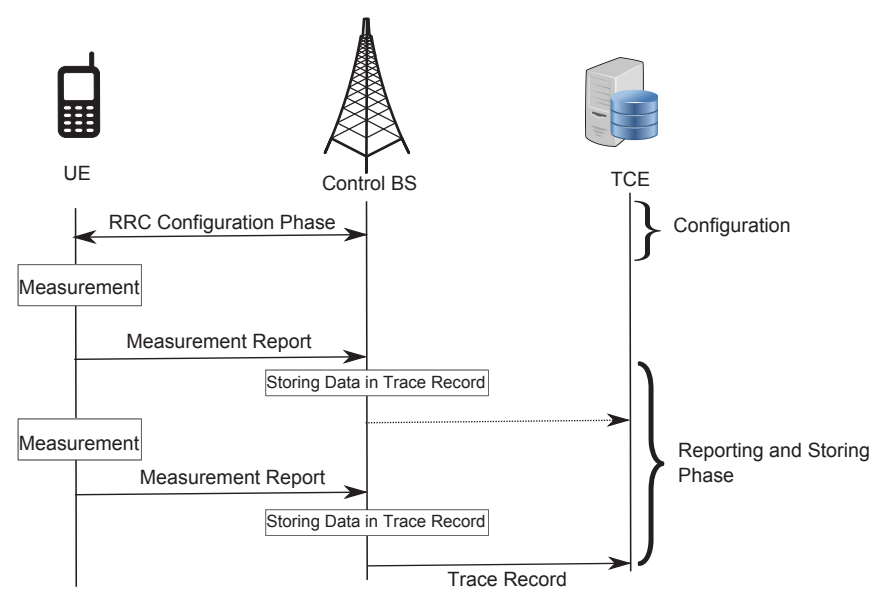

Fig. 2: Immediate MDT Signaling Flow

entity (TCE). TCE collects and stores the trace reports, which are subsequently processed to construct a MDT database. The trace record obtained from the reference scenario (i.e., faultfree) acts as a benchmark data and is used by the anomaly detection models to learn the network profile. These models are then employed to autonomously detect outage situations.

\section{B. Profiling}

In the profiling phase, the trace records are processed to extract the feature vector $\mathbf{O}$ corresponding to each MDT measurement. The measurements including reference signal 
Measurement

Location

Serving cell information

RSRP

RSRQ

Neighboring cell information

TABLE I: MDT Reported Measurements

received power and quality of the serving, as well as of the three strongest neighboring cells and the CQI are concatenated into a feature vector, $\mathbf{O}$, which is expressed as follows:

$$
\begin{array}{r}
\mathbf{O}=\left[R S R P_{S}, R S R P_{n 1}, R S R P_{n 2}, R S R P_{n 3}, R S R Q_{S},\right. \\
\left.R S R Q_{n 1}, R S R Q_{n 2}, R S R Q_{n 3}, C Q I\right],
\end{array}
$$

where the subscript $S$ and $n$ denotes the serving and neighboring cells, respectively. The observation vector, $\mathbf{O}$, is a 9-dimensional vector of numerical features that corresponds to one network measurement. To reduce the complexity of storage, processing and analysis this 9 -dimensional vector is embedded to three dimensions in the Euclidean space using the multi-dimensional scaling (MDS) method [33]. MDS provides a low dimensional embedding of the target measurement vectors $\mathbf{O}$ while preserving the pairwise distances amongst them. Given a $t \times t$ dissimilarity matrix $\boldsymbol{\Delta}^{\mathrm{X}}$ of the MDT dataset, MDS attempts to find $t$ data points $\psi_{1}, \ldots, \psi_{t}$ in $m$ dimensions, such that $\boldsymbol{\Delta}^{\Psi}$ is similar to $\boldsymbol{\Delta}^{\mathbf{X}}$. Classical MDS (CMDS) operates in Euclidean space and minimizes the following objective function

$$
\min _{\psi} \sum_{i=1}^{t} \sum_{j=1}^{t}\left(\phi_{i j}^{(X)}-\phi_{i j}^{(\Psi)}\right)^{2}
$$

where $\phi_{i j}^{(X)}=\left\|x_{i}-x_{j}\right\|^{2}$ and $\phi_{i j}^{(\Psi)}=\left\|\psi_{i}-\psi_{j}\right\|^{2}$. Equation (2) can be reduced to a simplified form by representing $\Delta^{X}$ in terms of a kernel matrix using

$$
\mathbf{X}^{T} \mathbf{X}=-\frac{1}{2} \mathbf{H} \boldsymbol{\Delta}^{\mathbf{X}} \mathbf{H},
$$

where $\mathbf{H}=\mathbf{I}-\frac{1}{t} \mathbf{e e}^{T}$ and $\mathbf{e}$ is a column vector of all 1 's. Hence (2) can be rewritten as

$$
\min _{\psi} \sum_{i=1}^{t} \sum_{j=1}^{t}\left(x_{i}^{T} x_{j}-\psi_{i}^{T} \psi_{j}\right)^{2} .
$$

As shown in [33], that $\boldsymbol{\Psi}$ can be obtained by solving $\boldsymbol{\Psi}=\sqrt{\boldsymbol{\Lambda}} \mathbf{V}^{T}$, where $\mathbf{V}$ and $\boldsymbol{\Lambda}$ are the matrices of top $m$ eigenvectors and the corresponding eigenvalues of $\mathbf{X}^{T} \mathbf{X}$, respectively. The $m$ dimensional embedding of the data points are the rows of $\sqrt{\Lambda} \mathbf{V}^{T}$, whereas the value of $m$ is chosen to be 3 in our case. The pre-processing of the network observation $\mathbf{O}^{e}$ using the MDS method has several advantages. In literature, the MDS technique has been widely used as a dimensionality reduction method [33]-[35] to transform highdimensional data into meaningful representation of reduced dimensionality. One of the problems with high-dimensional datasets is that in many cases not all the measured variables are "critical" for understanding the underlying phenomena. As shown in literature, that dimensionality reduction is a critical pre-processing step for the analysis of real-world datasets, since it mitigates the curse of dimensionality and other undesired properties of high-dimensional spaces [36]. MDS aims to achieve an optimal spatial configuration in a low dimensional space, such that distances in the new configuration (i.e., $\phi_{i j}^{(\Psi)}$ ) are close in value to the observed distances (i.e., $\left.\phi_{i j}^{(X)}\right)$. The spatial configurations help reveal a hidden structure that are not obvious from raw data matrices, allowing to explore the interrelationships of high-dimensional spaces. Given the growing complexity of the networks, particularly in case of SON, it is challenging to identify few measurements that accurately capture the behavior of the system. The MDS preprocessing of the network measurements allows to achieve a reduced representation that corresponds to intrinsic dimensionality of data. Consequently, the low-dimensional representation of network measurements facilitates data modelling and allows the anomaly detection algorithms to obtain better estimation of data density. As a result, the anomalous network measurements can be detected with higher accuracy, as discussed below. Moreover, unlike other dimensionality reduction methods such as principal component analysis (PCA) or linear discriminant analysis, MDS does not make an assumption of linear relationships between the variables, and hence applicable to wide variety of data.

In addition to network measurements, each MDT report is tagged with the location and time information as listed in Table I, which is used in conjunction with RSRP values to estimate the dominance or the coverage area of each target control BS in the network. The dominance map estimation is further used to autonomously localize the position of the outage control BS.

The next step after the pre-processing is to develop a reference database, $\mathbf{D}_{M}$, by storing the embedded measurements that represent the normal operation of the network. As shown in Fig. 3, this reference database is used by a state of the art anomaly detection algorithm to learn the "normal" network profile. The goal of this algorithm is to define an anomaly detection rule that can differentiate between normal and abnormal MDT measurements by computing a threshold ' $\varphi$ ' based on a dissimilarity measure ' $\mathcal{D}$ '. Thus, it can be treated as a binary classification problem which can formally be expressed as follows:

$$
f\left(x_{i}\right)= \begin{cases}\text { Normal, } & \text { if } \mathcal{D}\left(x_{i}, \mathbf{D}_{M}\right) \leq \varphi \\ \text { Anomalous, }, & \text { if } \mathcal{D}\left(x_{i}, \mathbf{D}_{M}\right)>\varphi\end{cases}
$$

Two state-of-the-art anomaly detection algorithms: $k$-NNAD and LOFAD are examined in our study. Anomalies, depending on their position in the MDS space, can be categorized as local or global anomalies. Local anomalies are localized to a small spatial region (i.e. local density) or a neighbourhood, whereas global anomalies are bounded to the entire dataset (i.e. global densities). $k$-NNAD computes a global dissimilarity measure $\mathcal{D}_{k-\text { NNAD }}$, which assigns a score to the test observation $x_{i}$ based on its distance from the $k^{\text {th }}$ nearest training point in the MDS space. On the other hand, LOFAD, instead of the distance measure, compares the local density of $x_{i}$ to its $k$ neighbours and correspondingly assigns a score. The correct profiling of the network behaviour is dependent on the 


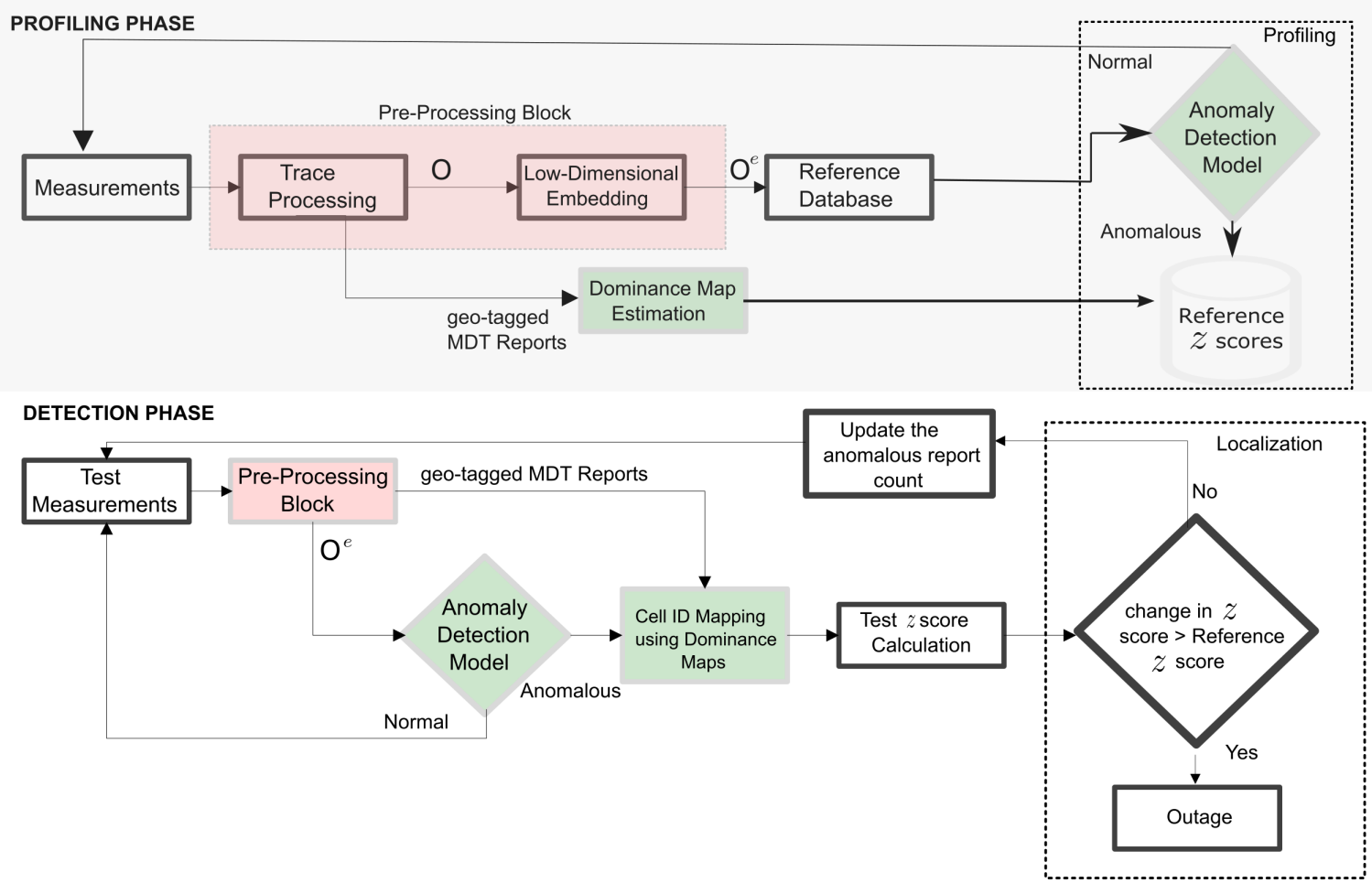

Fig. 3: An overview of profiling and detection phases in control COD framework

accuracy of the anomaly detection models. The two anomaly detection algorithms are briefly summarized as follows:

1) $k$-NNAD: Let $x_{i}$ be the test instance, and $k$ be the $k^{\text {th }}$ neighbor in the $\mathbf{D}_{M}$. To label $x_{i}$ as normal or abnormal, the $k$-NNAD computes a $\mathcal{D}_{k \text {-NNAD }}$ as

$$
\mathcal{D}_{k-\mathrm{NNAD}}\left(x_{i}, k, \mathbf{D}_{M}\right)=\frac{1}{N_{t r}} \sum_{i=1}^{N_{t r}} \mathcal{I}\left(d_{t} \leq d_{i}\right),
$$

where $N_{t r}=\left|\mathbf{D}_{M}\right|, d_{t}$ is the distance of $x_{i}$ from its $k^{t h}$ nearest neighbor, $d_{i}$ is the distance between $i$ and its $k^{\text {th }}$ nearest training object in $\mathbf{D}_{M}$, and $\mathcal{I}($.$) is an indicator function. The$ indicator function is activated as soon the condition $d_{t} \leq d_{i}$ is fulfilled. The expression in (6) represents a global densitybased anomaly detection score as proposed in [37]. The test measurement is marked as anomalous if it receives a score greater than the $\varphi$ value.

2) LOFAD: The LOFAD [38] tries to compare the local density, $\rho$, of the object to that of its $k$ neighbors. It constructs a local neighborhood of an instance $x_{i}$ and defines its distance to the $k^{\text {th }}$ nearest neighbor $N N\left(x_{i}, k\right)$ :

$$
d_{b}\left(x_{i}, k\right)=d\left(x_{i}, N N\left(x_{i}, k\right)\right)
$$

The $d_{b}\left(x_{i}, k\right)$ is used to construct a neighborhood $\mathcal{N}\left(x_{i}, k\right)$ by including all those points, which fulfills the criteria: $d\left(x_{i}, x_{j}\right) \leq d_{b}\left(x_{i}, k\right)$. Formally, reachability distance $d_{r}$ is defined to estimate the $\rho\left(x_{i}, k\right)$ as follows:

$$
d_{r}\left(x_{i}, k\right)=\max \left\{d_{b}\left(x_{j}, k\right), d\left(x_{j}, x_{i}\right)\right\}
$$

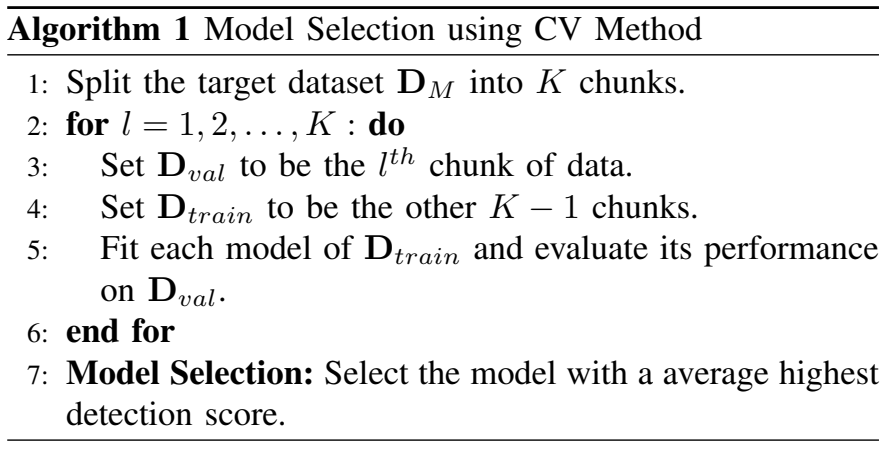

and $\rho$ can be defined as

$$
\rho\left(x_{i}, k\right)=\frac{\left|\mathcal{N}\left(x_{i}, k\right)\right|}{\sum_{x_{j} \in \mathcal{N}\left(x_{i}, k\right)} d_{r}\left(x_{i}, x_{j}, k\right)}
$$

The $d_{r}\left(x_{i}, x_{j}, k\right)$ ensures that instances that lie farther away from $x_{i}$ have less impact on $\rho\left(x_{i}, k\right)$. Finally the $\mathcal{D}$ can be calculated by comparing the $\rho$ of $x_{i}$ to its $\mathcal{N}\left(x_{i}, k\right)$, formally defined as:

$$
\mathcal{D}_{\text {LOFAD }}\left(x_{i}, k ; \mathbf{D}_{\text {train }}\right)=\frac{\sum_{x_{j} \in \mathcal{N}\left(x_{i}, k\right)} \frac{\rho\left(x_{j}, k\right)}{\rho\left(x_{i}, k\right)}}{\left|\mathcal{N}\left(x_{i}, k\right)\right|}
$$

$\mathcal{D}_{\text {LOFAD }}$ represents a local density-estimation score, a value close to 1 means $x_{i}$ has the same density as its neighbors. On the other hand, a significantly high $\mathcal{D}_{\text {LOFAD }}$ score is an indication of anomaly.

The parameter selection for $k$-NNAD and LOFAD is performed using a cross-validation (CV) method, as described in Algorithm 1. The $\mathbf{D}_{M}$ is divided into training $\mathbf{D}_{\text {train }}$ and validation dataset $\mathbf{D}_{\text {val }}$ using $K$-folds approach, where the 
value of $K$ is chosen to be 10 in our framework. To select the optimal model, each target detector is trained for different values of $k$ and the model achieving the average highest detection score is selected.

As shown in Fig. 3, the control COD during the profiling phase calculates the reference $z$-score for each target control BS in the network using the benchmark data. The $z$-score is calculated as follows: $z_{b}=\frac{\left|N_{b}-\mu_{n}\right|}{\sigma_{n}}$, where $N_{b}$ is the number of MDT reports labeled as anomalies by the anomaly detection model for the target control BS $b$, and variables $\mu_{n}$ and $\sigma_{n}$ are the mean and standard deviation anomaly scores of the neighboring cells. In the profiling phase, we also estimate the so called dominance area, i.e., for each cell, we define the area where its signal is the strongest. To do this, we exploit the location information tagged with each measurement report, which will then allow us to associate a cell and the corresponding $z$-scores as shown in Fig. 3. The calculation of the dominance area along with the corresponding reference $z$-score for each control BS in the network, would allow us to detect an outage control cell situation autonomously in the detection phase, as discussed in the following subsection.

\section{Detection and Localization Phase}

In the detection phase, the test measurements are preprocessed in a similar fashion as in the profiling phase. The embedded representation $\mathbf{V}^{e}$ is classified as normal or anomalous by the anomaly detection models. Subsequently, the geo-location of each report is correlated with the estimated dominance maps (i.e., profiled during the profiling phase) to establish its correct cell association. This is because as soon as the control cell outage situation is triggered in the network, the malfunctioning control BS becomes no longer available. Consequently, the dominance or the coverage area of the neighboring cells increases, to serve the affected area. Therefore, if only E-UTRAN cell global identification (ECGI) information is utilized to localize the outage control cell, the anomalous MDT reports within the target area, would erroneously be associated to its neighboring cells. The label assigned by the anomaly detection model to each measurement in conjunction with its estimated cell-ID is used to calculate the test $z$-score in the outage scenario. Finally, the control cell outage situation is detected and localized by observing the change in the $z$-score obtained for each control BS in the outage scenario compared to the reference scenario.

\section{Data COD via Heuristic ApProach}

Contrarily to the control COD, which is performed at the $\mathrm{OMC}$, the data COD is performed at each control BS. Hence, establishing the normal state of the control BS is a prerequisite for data COD. The data COD process is organized into the trigger and detection phases, as illustrated in Fig. 4. All UEs associated with the data BS report their RSPP statistic to their serving control BS, which is later used in the detection process.

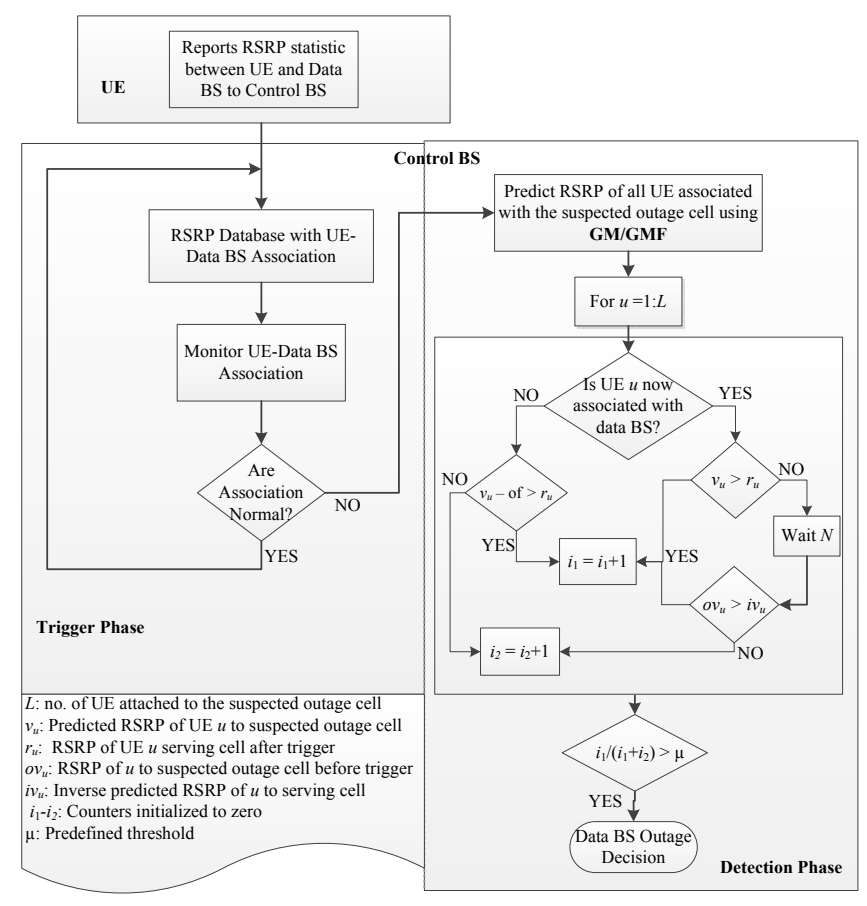

Fig. 4: Data cell outage detection flowchart

\section{A. Outage Trigger Phase}

The control BS receives a periodic update of the RSRP of the link between each UE and its associated data BS and stores this value in a database. As mentioned earlier in Section II-A, the control BS is responsible for managing UE connectivity, as well as radio specific functions, such as: 1) RRC connection, 2) configuration and measurement reporting, 3) cell handover and network controlled mobility. Consequently, the control BS is aware of any change in UE-data BS association, as a result of handover or radio link failure. The control BS is also aware of any state change in the UE, such as a change from active to idle state, idle to detached state and viceversa. Furthermore, the conditions for data BS to enter the sleep mode is known to the control BS. For example, the data BS could be allowed to enter the sleep mode if the number of active UEs is lower than a certain predefined threshold during the last scheduling time interval.

In the outage trigger phase, the control BS monitors the UEdata BS association and triggers the outage detection when it discovers irregularities in UE-data BS association. Irregularities in UE-data BS association occur when all UEs attached to a particular data BS change their association without any of the following: 1) prior handover initiation process, 2) change in state of all the UEs, 3) radio link failure notification from all the UEs, 4) the data BS going into sleeping mode.

\section{B. Outage Detection Phase}

Once the outage detection phase is triggered, the control BS can detect outage of the data BS by predicting the RSRP of all the UEs that were associated with it prior to the outage. We utilize the GM, which has been extensively used in handover, 
positioning and general forecasting algorithms [39]-[43], as prediction model.

1) GM Approach: In grey system theory, $G M(\bar{n}, \bar{m})$ denotes a grey model, where $\bar{n}$ is the order of the differential equation and $\bar{m}$ is the number of variables. Here we focus on $\operatorname{GM}(1,1)$, which is a widely used time series forecasting model. According to [39], the $G M(1,1)$ model can only be used on positive data sequences. Note that the RSRP values are always positive, hence, the grey model can be used to predict the next RSRP value from data points obtained in the database.

The three basic operations in grey prediction are: 1) the accumulated generating operation (AGO), 2) the inverse accumulated generating operation (IAGO), 3) grey modelling. By using AGO, an irregular raw data can be transformed into a regular data, which can be used to construct a model in grey differential equation. The non-negative RSRP data sequence of UE $u$ prior to the outage is denoted as

$$
r_{u}^{(0)}=\left(r_{u}^{(0)}(1), r_{u}^{(0)}(2), r_{u}^{(0)}(3), \ldots, r_{u}^{(0)}(n)\right), \quad \forall n \geq 4
$$

When the sequence given in (11) is subject to AGO, the following sequence, $r_{u}^{(1)}$ is obtained as

$$
r_{u}^{(1)}=\left(r_{u}^{(1)}(1), r_{u}^{(1)}(2), r_{u}^{(1)}(3), \ldots, r_{u}^{(1)}(n)\right), \quad \forall n \geq 4
$$

where

$$
r_{u}^{(1)}(c)=\sum_{i=1}^{c} r_{u}^{(0)}(i), \quad c=1,2,3, \ldots, n,
$$

which results in the grey differential equation given as

$$
\frac{d r_{u}^{(1)}(t)}{d t}+a r_{u}^{(1)}(t)=b
$$

The coefficients, $a$ and $b$, can be obtained by using least square method, as shown in (15):

$$
[a, b]^{T}=\left(B^{T} B\right)^{-1} B^{T} Y
$$

where

$$
\begin{aligned}
Y= & {\left[r_{u}^{(0)}(2), r_{u}^{(0)}(3), \ldots, r_{u}^{(0)}(n)\right]^{T}, } \\
B= & {\left[\begin{array}{cc}
-h^{(1)}(2) & 1 \\
-h^{(1)}(3) & 1 \\
\cdot & \cdot \\
\cdot & \cdot \\
-h^{(1)}(n) & 1
\end{array}\right], }
\end{aligned}
$$

and $h^{(1)}(c)=\alpha r_{u}^{(1)}(c)+(1-\alpha) r_{u}^{(1)}(c-1), c=2,3, \ldots, n$, $\alpha$ is the weighting factor.

Once $a$ and $b$ in (14) are obtained, the grey differential equation can be used to predict the value of $r_{u}$ at time instant $c+1$. The solution of $r_{u}^{(1)}(t)$ at time $c+1$, i.e. the AGO grey prediction model is expressed as

$$
\hat{r}_{u}^{(1)}(c+1)=\left[r_{u}^{(0)}(1)-\frac{b}{a}\right] e^{-a c}+\frac{b}{a}, \quad c=0,1, \ldots
$$

Consequently, the prediction value of the benchmark RSRP data at time $(c+1)$ can be calculated by an IAGO as

$$
\hat{r}_{u}^{(0)}(c+1)=\left[r_{u}^{(0)}(1)-\frac{b}{a}\right] e^{-a c}\left(1-e^{a}\right)
$$

2) GM Modification Using Fourier Series of Residual Error $(G M F)$ : According to [42] grey model prediction accuracy can be improved by the Fourier series of error residuals. Consider the $u^{t h}$ UE RSRP sequence, $r_{u}^{(0)}$ in (11) and its predicted values obtained from (18), then the error of the sequence $r_{u}^{(0)}$ can be expressed as

$$
\xi_{u}^{(0)}=\left(\xi_{u}^{(0)}(2), \xi_{u}^{(0)}(3), \ldots, \xi_{u}^{(0)}(n)\right)
$$

where

$$
\xi_{u}^{(0)}(c)=r_{u}^{(0)}(c)-\hat{r}_{u}^{(0)}(c), \forall c=2,3, \ldots, n .
$$

The error residuals given in (20) can be re-expressed in Fourier series in the following approximation

$$
\xi_{u}^{(0)}(c) \approx \frac{1}{2} a_{0}+\sum_{i=1}^{J}\left[a_{i} \cos \left(\frac{2 \pi i}{T} c\right)+b_{i} \sin \left(\frac{2 \pi i}{T} c\right)\right],
$$

$\forall c=2,3, \ldots, n$, where $T=n-1$ and $J=\left\lfloor\frac{n-1}{2}\right\rfloor-1$. Note that the expression in (21) can further be re-expressed as

$$
\xi_{u}^{(0)} \approx Q \mathcal{C}
$$

where

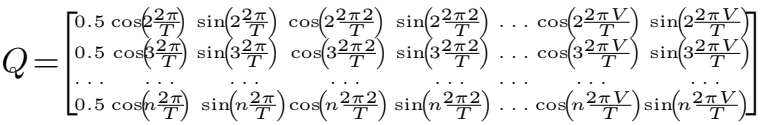

$$
\begin{aligned}
& \text { and } \quad \mathcal{C}=\left[\begin{array}{llllllll}
a_{0} & a_{1} & b_{1} & a_{2} & b_{2} & \ldots & a_{n} & b_{n}
\end{array}\right]^{T}
\end{aligned}
$$

Hence, $\mathcal{C}$ can be obtained by using the least square method to solve (22) as

$$
\mathcal{C}=\left(Q^{T} Q\right)^{-1} Q^{T} \xi_{u}^{(0)}
$$

The Fourier series correction is thus given according to [42] as

$$
\hat{\hat{r}}_{u}^{(0)}(c)=\hat{r}_{u}^{(0)}(c)-\hat{\xi}_{u}^{(0)}(c), \forall c=2,3, \ldots, n+1 .
$$

\section{Outage Decision}

Consider that the outage of data BS $d$, which has $L$ UEs attached prior to the outage trigger, is to be detected. Firstly, we define counters $i_{1}$ and $i_{2}$ and initialize their values to zero. The RSRP of the $L$ UEs that were previously attached to data BS, $d$, are predicted according to (18) or (25). Then, for each UE the control BS compares its predicted RSRP value, $v_{u}=\hat{r}_{u}^{(0)}(c+1) \approx \hat{\hat{r}}_{u}^{(0)}(c+1)$, with the RSRP after the trigger, $r_{u}=r_{u}^{(0)}(c+1)$. If afterwards outage is triggered, the $\mathrm{UE}, u$, is served by the control BS for data transmission and $v_{u}=\hat{r}_{u}^{(0)}(c+1) \approx \hat{\hat{r}}_{u}^{(0)}(c+1)>r_{u}-\Delta$, where $\Delta$ is the data cell range expansion offset, the counter, $i_{1}$, is incremented by 1 , since the UE should be associated with data BS, $d$, based on the prediction. Otherwise, the counter, $i_{2}$ is incremented by 1 . On the other hand, if another data BS is serving UE $u$, after the outage trigger and $v_{u} \approx \hat{r}_{u}^{(0)}(c+1) \approx \hat{\hat{r}}_{u}^{(0)}(c+1)>$ $r_{u}$, the counter, $i_{1}$, is incremented by 1 , otherwise an inverse prediction is performed on the RSRP to the serving data BS. The inverse prediction checks the RSRP to the data BS $d$ and the RSRP to the serving data BS after the trigger, i.e. data BS $\bar{d}$, at the point just before the trigger. The control BS waits for the prediction window size, $N$, and performs an inverse 
prediction on the RSRP of each of the UEs associated with data BS $\bar{d}$ to obtain the predicted RSRP prior to the trigger decision, $i v_{u}$. Thus, if the RSRP of the $u^{t h}$ UE to the serving data BS $(d)$ before trigger, $o v_{u}$, is such that $o v_{u}>i v_{u}$ the counter $i_{1}$ is incremented by 1 otherwise, the counter $i_{2}$ is incremented by 1 . The data cell outage is declared if the ratio $\frac{i_{1}}{i_{1}+i_{2}}>\mu$, where $\mu$ is a system predefined threshold.

\section{Reinforcement Learning Based Cell Outage COMPENSATION}

In this section, we provide a complete solution for COC. We consider, that the network has the capability to detect both data and control outages, through the solutions proposed in previous sections. We provide a solution capable of recovering both data and control outages. In particular, we consider an outage of a control BS in the control plane or data BS in the data plane. Hence, we optimize the coverage and capacity at the identified outage zone, by adjusting the gain of the antenna through the electrical tilt and the downlink transmission power of the surrounding control/data BS in the control/data plane. To implement this approach, we propose a RL scheme, which has the capability of making online decisions at each control/data BS, and of adapting to the evolution of the scenario, determined by factors like the UE mobility, shadowing and decisions made by the surrounding control/data BSs to the problem being solved.

\section{A. Reinforcement Learning}

The RL aims to learn from interactions to achieve a certain goal. The learner or decision maker is called agent, and it interacts continuously with the so called environment. The agent selects actions and the environment responds to those actions and evolves into new situations. In particular, the environment responds to the actions through rewards, numerical values, which the agent tries to maximize over time. The agent has to exploit what it already knows in order to obtain a reward, but it also has to explore in order to take better actions in the future. We assume that the environment is the wireless cellular scenario, with all its realistic characteristics, in terms of UEs mobility and activity patterns, and channel variations, while the agents are the control/data BSs.

Let $\mathcal{S}$ be the set of possible states of the environment $\mathcal{S}=\left\{s_{1}, s_{2}, \ldots, s_{n}\right\}$, and $\mathcal{A}$ be a set of possible actions $\mathcal{A}=\left\{a_{1}, a_{2}, \ldots, a_{q}\right\}$ that each agent may choose. The interactions between the multi-agent system and the environment at each time instant $t$ consist of the following sequence.

- control/data BS $i$ senses the state $s_{t}^{i}=s \in \mathcal{S}$.

- Based on $s$, control/data BS $i$ selects an action $a_{t}^{i}=a \in$ $\mathcal{A}$.

- As a result, the environment makes a transition to the new state $s_{t+1}^{i}=v \in S$.

- The transition to the state $v$ generates a reward $r_{t}^{i}=r \in$ $\Re$.

- The reward $r$ is fed back to the control/data BS and the process is repeated.

In the following we remove the notation indicating the specific agent $i$, for the sake of simplicity. At each time step, the agent implements a mapping from states to probabilities of selecting each possible action. This mapping is the agent's policy. The objective of each learning process is to find an optimal policy $\pi^{*}(s) \in \mathcal{A}$ for each $s$, to maximize some cumulative measure of the reward $r$ received over time. Almost all RL algorithms are based on estimating the so called value function, which is a function of the states estimating how good it is for an agent to be in a given state. The quantification of this is defined based on the expected future rewards. Of course, the rewards that an agent can expect to receive in the future depend on what actions it will take. As a result, the value of a state $s$ under a policy $\pi$, and denoted $\mathcal{V}^{\pi}(s)$, is the expected return when starting in $s$ and following $\pi$ thereafter.

$$
\mathcal{V}^{\pi}(s)=\mathbb{E}\left\{\sum_{t=0}^{\infty} \gamma^{t} r\left(s_{t}, \pi(s)\right) \mid s_{t}=s\right\}
$$

where $\mathbb{E}$ stands for the expectation operator and $0 \leq \gamma<1$ is a discount factor. Considering the extreme complexity of the dynamics of the complete wireless cellular environments, where the UEs move around the scenario according to random mobility models, the channel is affected by path loss, fading and shadowing, and the activity of UEs is again determined by random processes, we are not able to rely on a model of the environment's dynamic to solve this maximization problem. A solution is then to take advantage of the theory of RL and in particular of the so called temporal difference (TD) learning approaches. These kinds of methods are able to learn directly from experience, without a model of the environment's dynamics. Among the literature of TD learning schemes, we select the AC approach to adjust the downlink transmission power levels of the control/data BSs surrounding the outage zone, as it is one of the most representative TD schemes and it is not computationally complex. TD learning schemes can be proven to converge to optimal solutions, in stationary scenarios with only one decision maker, even though in practice they have been shown to provide successful results also in multi agent scenarios [44]. The proof of convergence of the learning algorithm is guaranteed by the Bellman optimality criterion [45]. Our solution is proposed for a multi-agent system where each agent self adapts based on an $\mathrm{AC}$ algorithm and the performances of each control/data BS is affected by the actions of the others in terms of inter cell interference.

\section{B. Actor Critic (AC)}

AC method is a TD method that has a separate memory structure to represent the policy independently of the value function. The policy structure is known as the actor, since it is used to select the actions, while the estimated value function is known as the critic. The critic learns and critiques whatever policy is currently being followed by the actor and takes the form of a TD error $\delta$, which is used to determine if $a_{t}$ was a good action or not. $\delta$ is a scalar signal, which is the output of the critic and drives the learning procedure. After each action selection, the critic evaluates the new state to determine whether things have gone better or worse than expected, as it is defined by the TD error:

$$
\delta_{t}=r_{t}+\gamma \mathcal{V}_{t}\left(s_{t+1}\right)-\mathcal{V}_{t}\left(s_{t}\right)
$$


where $\mathcal{V}$ is the current value function implemented by the critic, to evaluate the action $a_{t}$ taken in $s_{t}$. If the TD error is positive, it suggests that the tendency to select $a_{t}$ should be strengthened for the future, whereas if the TD error is negative, it suggests the tendency should be weakened. We identify this tendency with a preference function $P\left(s_{t}, a_{t}\right)$, which indicates the tendency or preference to select a certain action in a certain state. Then the strengthening or weakening described above can be implemented by increasing or decreasing $P\left(s_{t}, a_{t}\right)$ by

$$
P\left(s_{t}, a_{t}\right) \leftarrow P\left(s_{t}, a_{t}\right)+\beta \delta_{t}
$$

where $\beta$ is a positive learning parameter. This is the most simple implementation of a AC algorithm. The variation that we consider for implementation, is to add different weights to different actions, for example based on the probability of selecting action $a_{t}$ in state $s_{t}$, i.e. $\pi\left(s_{t}, a_{t}\right)$, which results in the following update rule:

$$
P\left(s_{t}, a_{t}\right) \leftarrow P\left(s_{t}, a_{t}\right)+\beta \delta_{t}\left(1-\pi\left(s_{t}, a_{t}\right)\right)
$$

In this implementation, $\mathrm{AC}$ directly implements the Boltzmann exploration method to select actions as follows:

$$
\pi\left(s_{t}, a_{t}\right)=\frac{e^{P\left(s_{t}, a_{t}\right)}}{\sum_{a_{t} \in \mathcal{A}} e^{P\left(s_{t}, a_{t}\right) / \tau}}
$$

This means the probability to select an action $a$ in state $s$ at time $t$ depends on the temperature parameter $\tau$, and on the preference values $P\left(s_{t}, a_{t}\right)$ at time $t$. In this kind of exploration, actions that seem more promising, because of higher preference values, have a higher probability of being selected.

1) $A C-R L$ for $C O C$ : In order to design the $\mathrm{AC}$ algorithm to implement the automatic transmission power and antenna tilt adjustment for the $\mathrm{COC}$, while mitigating the generated interference and improving the capacity of the zone, we need to define the state and action spaces and the reward function.

- State: The state is defined based on the result of the scheduling scheme, which defines: (1) the allocation of UEs to RBs (i.e, $R B_{1}, R B_{2}, \ldots, R B_{R}$ to the $N$ UEs in the plane), (2) the values of CQI of each UE in the corresponding $\mathrm{RB}$.

- Actions: The set of eligible actions are:

- The finite set of downlink transmission power levels, which can be allocated to the RBs assigned to the UEs. The selected values are: 0 to $23 \mathrm{dBm}$ per RB for data $\mathrm{BS}$ and 0 to $46 \mathrm{dBm}$ per RB for control BS; each with $0.5 \mathrm{dBm}$ granularity.

- The finite set of available tilt values, which can be assigned to the gain of the vertical plane of the antenna model. Those values range from 0 to $15^{\circ}$ with $0.5^{\circ}$ granularity.

- Reward: The reward function is defined based on the signal to interference and noise ratio (SINR) of the UEs as follows:

\begin{tabular}{|c|c|}
\hline Parameter & Value \\
\hline Tx Power Control BS & $46 \mathrm{dBm}$ \\
\hline Tx Power Data BS & $23 \mathrm{dBm}$ \\
\hline Path loss model & Friis spectrum propagation \\
\hline Mobility model & pedestrian, speed $3 \mathrm{kmph}, 60 \mathrm{kmph}$ \\
\hline UE distribution & Uniform random distribution \\
\hline Scheduler & FFR \\
\hline Shadow Fading & Log-normal, std = 2-10dB \\
\hline AMC model & 4-QAM, 16-QAM, 64 QAM \\
\hline Macro cell layout & radius:500 $\mathrm{m}$ \\
\hline Bandwidth per plane & $5 \mathrm{MHz}$ \\
\hline No. of RBs & 25; RBs per RBG:2 \\
\hline Antenna gain (Normal Scenario) & $18 \mathrm{dBi}$ \\
\hline Antenna gain (Outage Scenario) & $-50 \mathrm{dBi}$ \\
\hline MDT reporting interval & $240 \mathrm{~ms}$ \\
\hline Minimal sensible signal strength & $-107.5 \mathrm{dBm}$ \\
\hline Detection threshold $\mu$ & 0.5 \\
\hline Detection window size $N$ & 10 \\
\hline Grey weighting factor $\alpha$ & 0.5 \\
\hline SINR threshold & $-6 \mathrm{~dB}$ \\
\hline Actions (Control BS power) & $\begin{array}{l}0-46 \mathrm{dBm} \text { per RB: } \\
\text { Granularity } 0.5 \mathrm{dBm}\end{array}$ \\
\hline Actions (Data BS power) & $\begin{array}{l}0-23 \mathrm{dBm} \text { per RB: } \\
\text { Granularity } 0.5 \mathrm{dBm}\end{array}$ \\
\hline Actions (tilt) & $0^{\circ}-15^{\circ}:$ Granularity $0.5^{\circ}$ \\
\hline Parameters $\tau, \beta, \gamma$ & $0.1,0.5,0.98$ \\
\hline Simulation time & 10 minutes \\
\hline
\end{tabular}

$$
r\left(s_{t}, a_{t}\right)=\left\{\begin{array}{lc}
1, & \text { if } S I N R \geq-6 \\
0, & \text { otherwise }
\end{array},\right.
$$

TABLE II: Simulation Parameters.

where the threshold is set in order to support the lowest modulation and coding scheme (MCS) [9] for long term evolution (LTE).

In order to mitigate the inter-cell interference that the adjustment of the neighboring control/data BS power level may generate in the outage zone, frequency bandwidth $\mathcal{B}=$ $\left\{f_{1}, \ldots, f_{7}\right\}$ is reserved for the UEs moving in the outage zone while $f$ serves UEs in each of neighbouring cells to the outage BS, as illustrated in Fig. 1 (FFR2).

The learning algorithm is executed every $1 \mathrm{~ms}$, which is also the smallest scheduling time interval in LTE. Hence, the solution of the learning algorithm can be applied when allocating UEs every time scheduling is performed. The execution of the learning algorithm at TTI resolution increases the speed with which the fault and the outage are recovered. However, it is possible to execute the algorithm less often, to reduce the computational complexity, but this will increase the time the system suffers from the outage generated by the fault. In practice, the operator implementing the algorithm can set the frequency of execution to the preferred value based on trading off the implementation criteria considered more important. We recommend that the learning algorithm must be executed with a low periodicity during the emergency situation, to speed up the recovery when many users have seen their quality off experience (QoE) compromised, and with a higher periodicity when the outage is about to be fully recovered. The CQI is obtained from the spectral efficiency $(\eta)$ provided in [46]. A high level vision of the COC algorithm is illustrated in Fig. 1.

\section{Simulation Results And Discussions}

\section{A. Simulation Scenario}

In this section, we demonstrate the performance of our HetNet COM framework by presenting simulation results for 


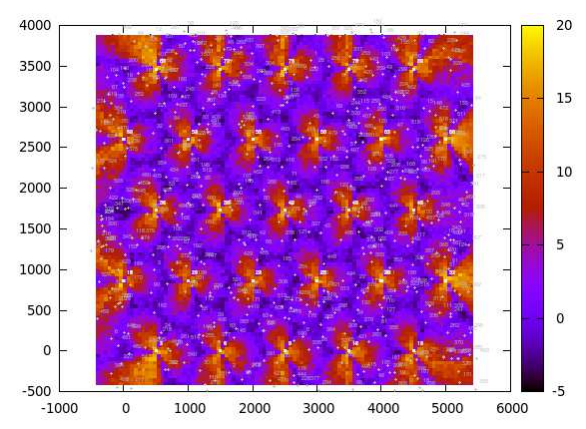

(a) Reference Scenario

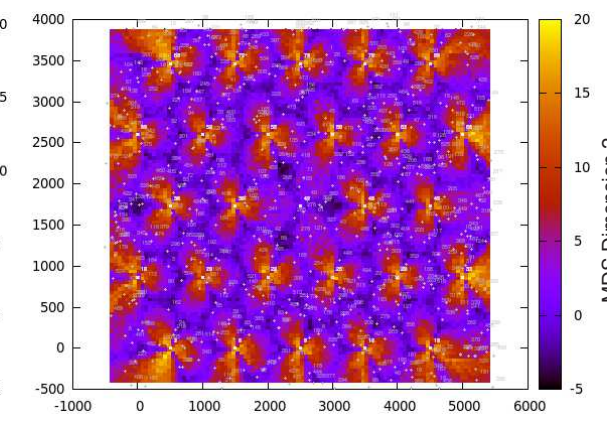

(b) Control Cell Outage Scenario

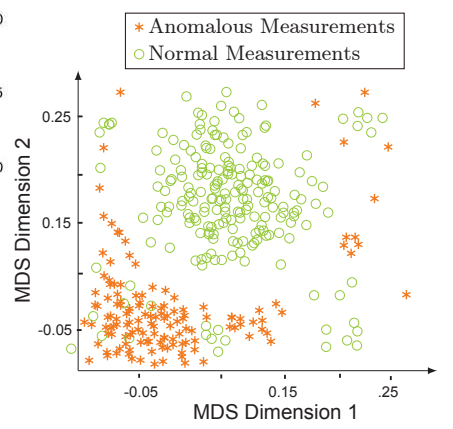

(c) MDS Embedding of MDT Reports

Fig. 5: (a) SINR plot of reference scenario (b) SINR plot of control cell outage scenario where antenna gain of cell 11 is attenuated to $-50 \mathrm{dBi}$ (c) MDT measurements in the embedded space classified into normal and anomalous categories by $k$-NNAD

the COD and COC algorithms. We consider a HetNet architecture where the control and data BSs operate on separate frequency carriers. Each operation mode occupies $5 \mathrm{MHz}$ of channel bandwidth. The scenario that we set up consists of 27 macro/control BSs with $U_{m}=20$ UEs per control BS, and $F B=5$ femtocell blocks per control BS, each one with $l=40$ apartments, $t=1$ floor, $d=0.2$ small/data BS deployment ratio as per [47], and $c=0.5$ data $\mathrm{BS}$ activation ratio, which results in 20 data BSs per control BS. Also, there are $U_{f}$ UEs per data BS in the scenario. The principle of UE association is such that each UE associates with a control BS for signalling transmission. Furthermore, each UE associates with a data BS for data transmission if its RSRP from the data BS exceeds that of the control BS, otherwise, it associates with a control BS for both data and control signalling transmission. The parameters used in the simulations, for the cellular scenario, control and data COD algorithms, and the COC algorithm, are given in Table II.

\section{B. $C O D$}

We simulate the reference scenario to profile the normal network operation, in which all UEs are configured to report the MDT measurements on the control plane. In addition, UEs that are associated with the data cells are also configured to periodically report their RSRP statistics (in the data plane) to their serving control BS. A control cell outage scenario is simulated by attenuating the antenna gain of control BS 11 in the network to $-50 \mathrm{dBi}$ for a duration of three minutes. The total simulation run time was 10 minutes for each scenario. Likewise, for the data cell outage, we focus of on control BS 10 and attenuate the antenna gain of data BS 11 within its coverage to $-50 \mathrm{dBi}$. The respective SINR plots of the reference and outage scenario in the control plane is shown in Fig. 5. The collected measurements are processed by our proposed control and data COD frameworks, as shown in Figs. 3 and 4, respectively.

1) Control COD: Local and global anomaly detection approaches, namely LOFAD and $k$-NNAD, have been compared to select the final model for profiling the network behaviour. The receiver operating characteristic (ROC) curves [48] plot the true positive rate or detection rate (DR) (i.e., a percentage of anomalous measurements correctly classified as anomalies) against the false positive rate (FPR) (i.e., a percentage of normal cell measurements classified as anomalies). The ROC curves are generated by plotting the DR against FPR by varying the $\varphi$ for each model until a DR value reaches $100 \%$. To access the performance of the target algorithms, a standard performance metric named as area under ROC (AUC) curve is employed. To select the optimal model for each anomaly detector, a parameter search (i.e. $k=1,2, \ldots 30$ ) is performed using Algorithm 1. The final values of $k$ are found to be 20 and 8 for $k$-NNAD and LOFAD, respectively.

It has been observed that measurements that conform to the normal behavior of network operations, when projected to an embedded space group themselves into a large cluster. On the other hand, when the measurements belonging to outage control cell scenario are projected to the embedded space, they lie far from the dense cluster of normal measurements, as depicted in Fig. 5c. The reason is that MDS embedding of the measurements maximize the variance between the data points and consequently dissimilar points are projected far from each other. This embedded representation of measurements reveals a hidden structure of data. In particular, this is helpful for the density based anomaly detection models as employed in this study, to learn an effective anomaly detection rule. Since, such models assume that anomalous data lie in low density regions, the embedded representation of the data aids in establishing a boundary between high and low density regions. Consequently, an effective anomaly detection rule can be learned. $k$-NNAD based global profiling technique, which relies on the global density estimation procedures, outperforms local density estimation method LOFAD, since the anomalous measurements obtained from the outage scenario largely act as global anomalies. Moreover, some of the normal measurements also form small micro clusters. This is due to exceptionally good RSRP values reported by the mobile terminals while they are in close proximity to the serving control BS. However, LOFAD treats them as local anomalies. Additionally, the measurements obtained from the cell edges show similarity with data samples that correspond to the outage scenario. Hence, in the embedded space they are projected close to the samples that corresponds to abnormal measurements. From a classification perspective, the target models wrongly classify such measurements as belonging to an outage control cell scenario. However, from a 


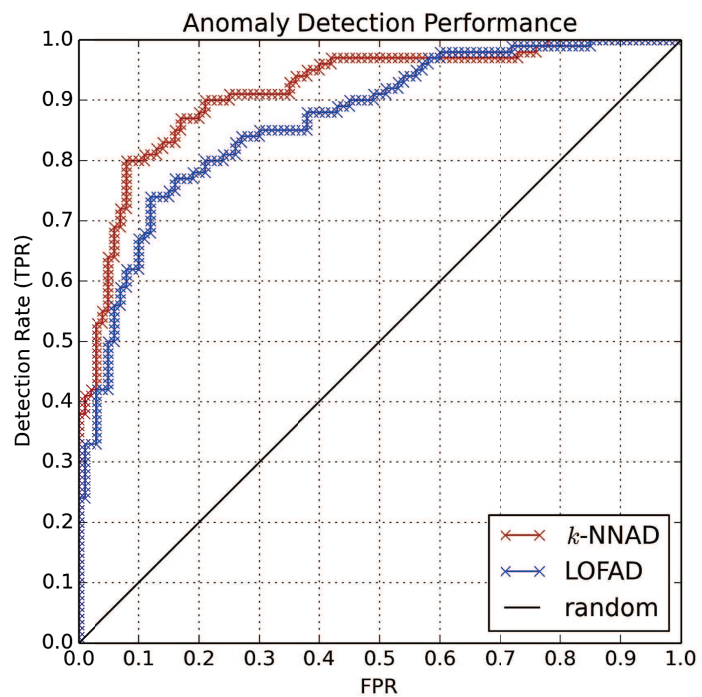

Fig. 6: ROC Curve of $k$-NNAD based profiling technique

SON perspective, identification of such abnormality indicates a weak coverage problem and can be used to trigger automated actions for coverage optimization. Similarly, some of the UE generated measurements, as a result of radio link failure, are also treated as anomalies. Fig. 6 shows that $k$-NNAD achieves $80 \%$ DR, which is $15 \%$ higher than LOFAD at a false alarm rate of $10 \%$. As shown in Table III, the AUC value achieved by $k$-NNAD and LOFAD are 0.91 and 0.85 , respectively, which shows the superiority of global anomaly detection methods over local approaches for profiling the network behavior.

We use $k$-NNAD as our target model to calculate the $z$ score for each control BS, separately, for reference and control cell outage scenarios, as shown in Fig. 7. It can be observed that even the cells which are not in outage receive a $z$-score, since a fraction of the UE reported measurements, belonging to their dominance areas, are identified as anomalies due to the reasons discussed earlier. Therefore, to classify a cell as in outage, each control BS must collect a minimum number of anomalous reports (i.e., $N_{b}$ ) to achieve a significantly higher $z$-score compared to the rest of cells. We collect approximately 4800 measurements in one minute from each cell, since our scenario is characterized by 20 uniformly distributed UEs that send reports with a periodicity of $240 \mathrm{~ms}$.

The $z$-scores shown in Fig. 7 for each control cell, are normalized by the total number of measurements obtained every minute. It can be observed that the outage cell (i.e., control cell 11) achieves a highest percentage $z$-score of 0.449 ( $N_{b}=3520$, with mean and standard deviation values of neighboring cells as 500 and 140, respectively), which is above our heuristically set threshold of 0.3 . The test $z$-score of the outage cell deviates significantly from its reference $z$-score of 0.09 , and it can be detected easily. Likewise, the delay value can increase or decrease depending on the UE density in the target cell.

2) Data COD: Fig. 8 illustrates the performance of our data COD framework in terms of the DR. In Fig. 8a, we compare the performance of the GM and GMF, which are obtained from (18) and (25), respectively, with the cooperative

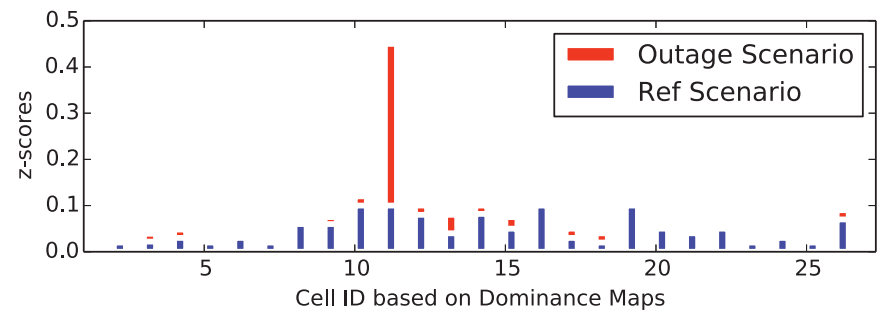

Fig. 7: Cell Dominance areas versus $z$-scores for Localization of control cell outage

\begin{tabular}{c|c|c}
{$[\mathrm{t}]$ Model } & Approach & AUC score \\
\hline$k$-NNAD & Global & 0.91 \\
\hline LOFAD & Local & 0.85 \\
\hline
\end{tabular}

TABLE III: Performance of target anomaly detection models for control COD

COD approach of [5], by plotting their DR against the data cell UE density, $U_{f}$, and for shadowing fading standard deviation of, $\sigma=2$ and $10 \mathrm{~dB}$. For the cooperative COD scheme, we consider the coopeartive range as the coverage area of each control BS. We observe that the proposed schemes, which are also independent of the cooperative range, outperform the cooperative COD scheme of [5]. We observe that the GMF scheme outperforms the GM as expected, since the former utilizes the prediction error in the later to improve its performance. Fig. 8a clearly shows that increasing the UE density increases the DR. This is due to the fact that increasing UE density enables a better spatial correlation. Fig. 8b depicts the DR for various data BS power levels and a data cell UE density of $U_{f}=3(/ 100 \mathrm{~m} \times 100 \mathrm{~m})$. The result shows that low data BS transmission power results in degradation of the DR, while increasing the transmission power leads to an improvement in the DR. This is because when the data BS transmission power increases, it becomes easier to distinguish between the predicted RSRP statistics of the outage case and normal case. We also observe in Figs. $8 \mathrm{a}$ and $8 \mathrm{~b}$ that the DR becomes lower with larger shadowing fading standard deviation $\sigma$. This is because a high $\sigma$ means a severe shadowing fading, which leads to a more random RSRP statistics.

Figs. 9a and 9b investigate the impact of the predefined threshold $\mu$ and prediction window size $\mathcal{N}$, respectively, on the DR. We observe in Fig. 9a that the highest DR is obtained by setting $\mu=0.5$. This setting implies that the RSRP prediction of more than half of the UEs that were associated with the data BS whose outage is being detected, i.e. $d$, must indicate the existence of an outage, before $d$ can be declared to be in outage. The stepwise shaped plot is obtained since the number of UEs must be an integer value. We also observe that there is not much degradation in DR until $\mu>0.67$, which implies more than two-third of UEs that were associated with $d$ must indicate the existence of an outage. In Fig. 9b, we observe that increasing the prediction window size above the required minimal $(\mathcal{N}=4)$ leads to an increase in DR up to a point where any further increase in $\mathcal{N}$ has no impact on the DR. Fig. 9b further shows that increasing $\mathcal{N}$ has more impact on 


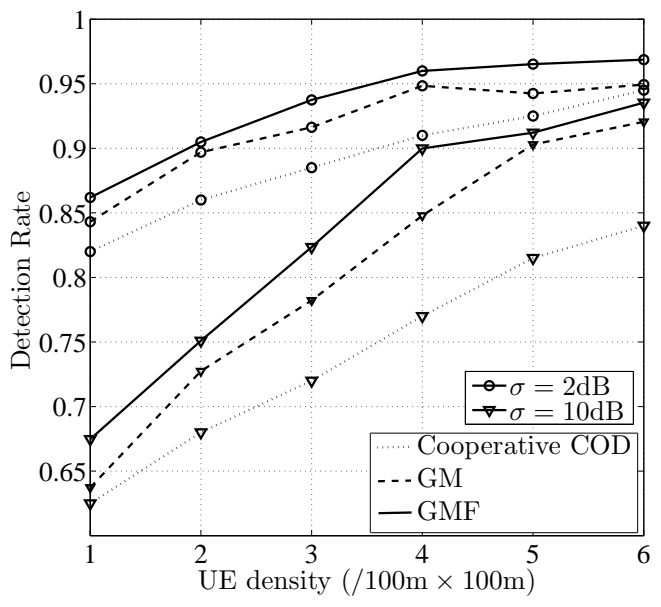

(a) GM vs GMF

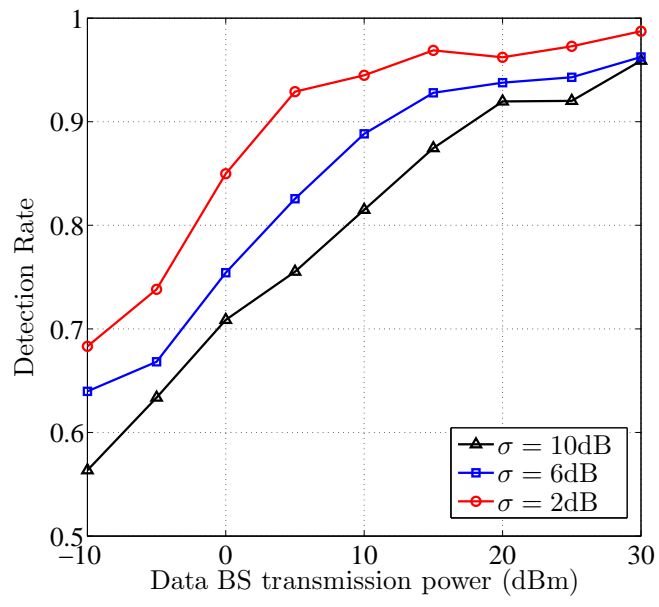

(b) Effect of data BS transmission power on DR.

Fig. 8: Performance of data COD framework

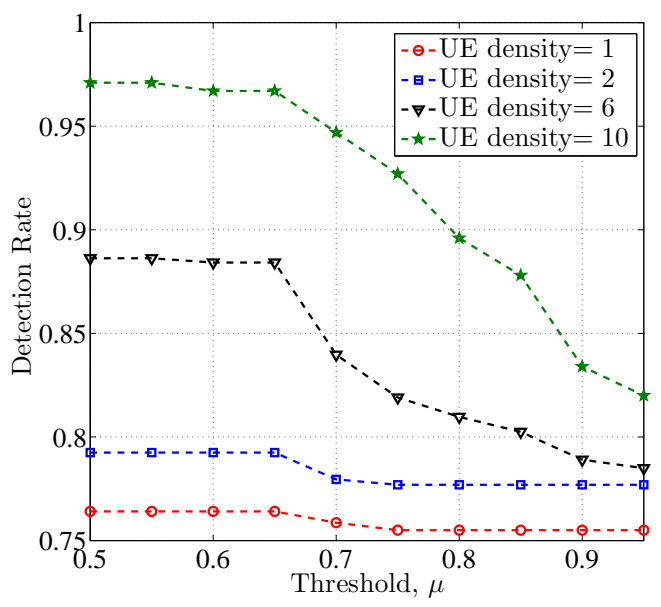

(a) Effect of threshold setting on DR

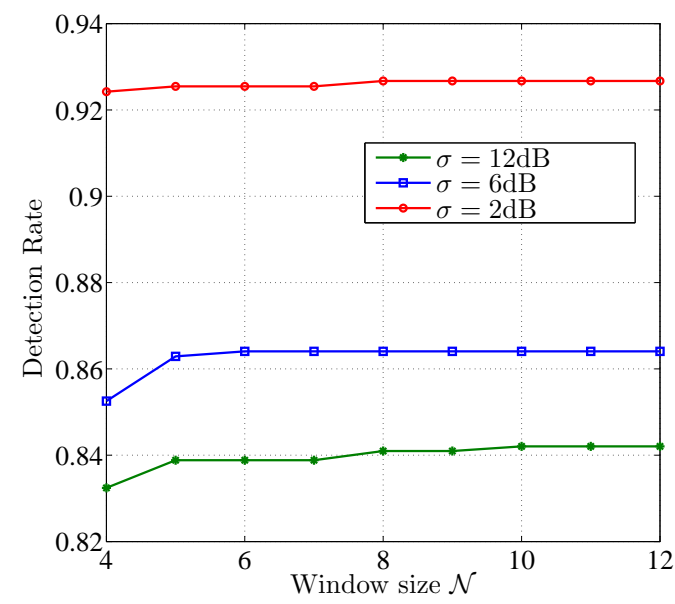

(b) Effect of window size on DR.

Fig. 9: Effect of data COD algorithm parameters on DR

the DR for larger shadow fading standard deviation, $\sigma$. This is because of the lower randomness in RSRP statistics when $\sigma$ is low; hence a low value of $\mathcal{N}$ is required to obtain the highest attainable DR, which is the contrary for higher $\sigma$ where a higher value of $\mathcal{N}$ is required.

\section{C. $\mathrm{COC}$}

Here we consider that outage occurs when a data BS 11 in control BS 10 fails to provide service to its associated UEs. We assume that the data COD framework in the previous subsection has already detected the problem and we therefore focus on the COC solution. Here, the neighboring cells are in charge of adjusting their power transmission, and antenna tilt in order to fill the coverage gap. We start by analyzing the behavior of a UE attached to the faulty data BS. We observe that once the COC algorithm is operational, the UE gets associated to one of the neighboring data BSs, which we refer to as the compensating cell. The CQI associated to the UE in this moment is zero. The scheduling scheme is implemented to minimize the interference generated from compensating cells, by reserving a certain resource block group (RBG) to the UE. This information is shared with neighbor data BSs through the X2 interface. We refer to the AC algorithm in Section V, where both the Tx power and antenna tilt are adjusted, as the $\mathrm{AC}(\mathrm{p}+\theta)$.

Fig. 10, depicts the time evolution of the SINR of the UE from the instant in which the data cell outage is detected and the COC algorithm starts working, till when it is recovered and correctly associated to the compensating cell. We show the behavior of the algorithm for both pedestrian $(3 \mathrm{kmph})$ and vehicular $(60 \mathrm{kmph})$ scenarios. We observe that in both cases the COC algorithm takes advantage of its capability of making online decisions, properly adapting to the evolution of the environment, even when it is varying quickly, as it happens in vehicular settings. We assume that the UE is recovered when its SINR is above the threshold, which is set to $-6 \mathrm{~dB}$ as explained in Section V-B1. The behavior followed by the $\mathrm{AC}(\mathrm{p}+\theta)$ scheme is such that, during the first part of 


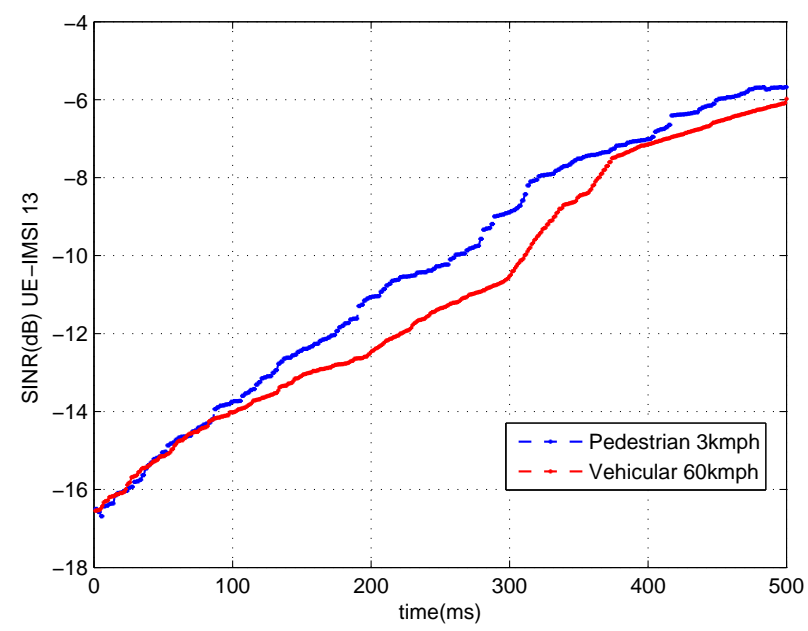

Fig. 10: Time evolution of the average SINR of UE 13, which is recovered from outage by the COC solution based on AC.

the simulation, it learns the proper policy to recover the UE from outage through interactions with the environment. After approximately $450-500 \mathrm{msec}$, for the pedestrian and vehicular cases, respectively, the UE SINR reaches the threshold, and maintains higher values during the rest of the simulation. Since the correct policy has been learnt, it can be maintained independently of the variations in the scenario, such as UE mobility, random data BS activity factor, etc. Fig. 10 further shows that the performance in the high mobility (vehicular) is worse compared to the pedestrian case, hence, a slightly longer time is required to recover from outage in the high mobility

Fig. 11 shows the performances of our COC framework in terms of SINR, RSRQ and RSRP of the UEs, represented by means of the cumulative distribution function (CDF), and compared to the case where no COC scheme is implemented, i.e. NO COC in the figures, and to the state of the art benchmark, discussed in [49] and based on fuzzy logic theory. Fig. 11a represents the CDF of the SINR of the UEs in the scenario, after convergence of the learning approach. It shows that the proposed $\mathrm{AC}(\mathrm{p}+\theta)$ scheme performs better than both the NO COC option and the fuzzy logic scheme. Fig. 11b shows the CDF of the RSRQ of the UEs in the scenario, after convergence of the learning approach. Here, again we observe that the $\mathrm{AC}(\mathrm{p}+\theta)$ scheme provides better results than the Fuzzy Logic approach and the NO COC scheme. Finally, Fig. 11c shows that the proposed $\mathrm{AC}(\mathrm{p}+\theta)$ provides better results also in terms of RSRP than the fuzzy logic based and the NO COC schemes.

\section{COM Framework Cost:}

Here we give some insight on the cost associated with implementing the proposed COM framework. The cost metric encompasses the complexity in analysis, processing, storage and signaling overhead. A large part of the cost associated with our proposed COM framework lies in the control COD, as it requires large number users and measurement reports to achieve a high DR. This cost has been substantially re- duced in our control COD solution by employing a MDS method, which embeds each network measurement from a 9dimensional feature vector into three dimensions in Euclidean space. Furthermore, the data COD solution in our framework is implemented in a fully distributed fashion at each control BS, thus significantly reducing the implementation cost of the framework. COC solutions on the other hand are implemented in semi-distributed fashion by dividing the system into clusters, where each cluster contains the outage cell and its neighbors, thereby reducing the signaling overhead and processing cost of the framework.

\section{CONCLUSION}

In this paper, we have presented a novel cell outage management (COM) framework for HetNets with split control and data planes. Two distinct cell outage detection (COD) algorithms have been proposed taking into account expected large number of UEs in the control cells and small number of UEs in the data cells. For control COD, we have utilized the large scale data gathering of MDT reports, as recently standardized by $3 \mathrm{GPP}$ in release 10 . The solution exploits multidimensional scaling techniques to reduce the complexity of data processing while retaining pertinent information to develop training models to reliably apply anomaly detection techniques. Furthermore, within the control COD, we have compared the performance of two anomaly detecting algorithms, i.e., $k$-NNAD and LOFAD. We found out that $k$-NNAD, which is a global anomaly detection model, achieved a higher detection accuracy of up to $94 \%$ compared to LOFAD, which adopts a local approach for classifying abnormal measurement. On the other hand, for data cell outage, we have utilized a heuristic grey-prediction approach, which can reliably work despite of the small number of UEs in the data cells by exploiting the information stemming from the fact that the control BS manages the UE connectivity to the data BS within its coverage. The simulation results have shown that both control and data COD schemes can detect control and data cell outages, respectively, in a reliable manner. In addition, we have proposed an AC based RL algorithm, which can be applied for both the control and data cell outage compensation (COC). The proposed COC algorithm solution was based on optimizing the coverage and capacity of the identified outage zone, by adjusting the gains of the antennas through electrical tilt, and downlink transmission power of the surrounding BSs in that plane. Simulation results have shown that the AC-RL algorithm can recover all UEs from outage within a very short time.

\section{ACKNOWLEDGMENT}

This work was made possible by NPRP grant No. 5-10472437 from the Qatar National Research Fund (a member of The Qatar Foundation). The statements made herein are solely the responsibility of the authors. We would like to acknowledge the support of the University of Surrey 5GIC members for this work. 


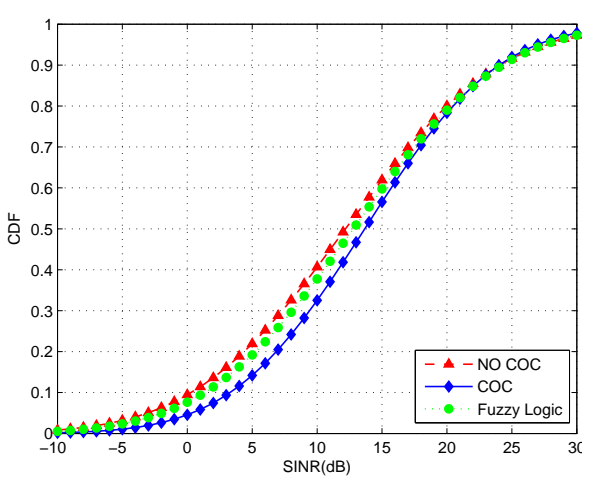

(a) CDF of the SINR of the serving cells.

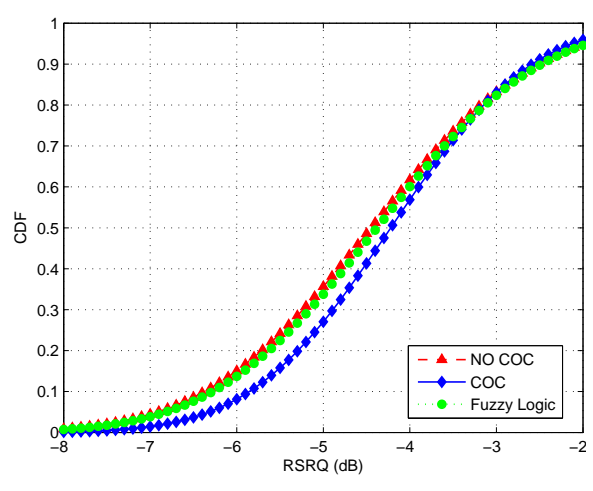

(b) CDF of the RSRQ of the serving cells.

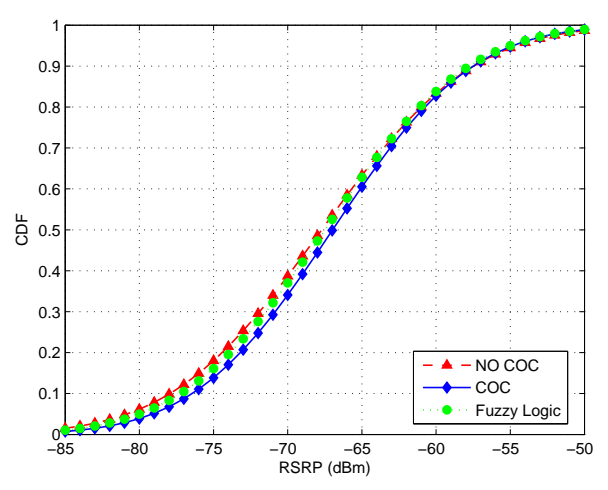

(c) CDF of the RSRP of the serving cells.

Fig. 11: Comparison of the CDF of SINR, RSRQ and RSRP of the COC with Fuzzy Logic, and NO COC

\section{REFERENCES}

[1] J. L. Van den Berg, R. Litjens, A. Eisenbltter, M. Amirijoo, O. Linnell, C. Blondia, T. Krner, N. Scully, J. Oszmianski and L. C. Schmelz, "Self-Organisation in Future Mobile Communication Networks," in ICT Mobile Summit, Sweden, 2008.

[2] O. G. Aliu, A. Imran, M. A. Imran, and B. G. Evans, "A Survey of Self Organisation in Future Cellular Networks," IEEE Commun. Surveys Tuts., vol. 15, no. 1, pp. 336-361, 2013.

[3] R. Combes, Z. Altman, and E. Altman, "Self-Organization in Wireless Networks: A Flow-Level Perspective," in IEEE INFOCOM, Mar. 2012, pp. 2946-2950.

[4] C. Mueller, M. Kaschub, C. Blankenhorn, and S. Wanke, "A Cell Outage Detection Algorithm Using Neighbor Cell List Reports," in International Workshop on Self-Organizing Systems, 2008, pp. 218-229.

[5] W. Wang, J. Zhang, and Q. Zhang, "Cooperative Cell Outage Detection in Self-Organizing Femtocell Networks," in IEEE INFOCOM 2013, Apr. 2013, pp. 782-790.

[6] 3GPP TS 32.541, "3rd Generation Partnership Project; Technical Specification Group Services and System Aspects; Telecommunications Management; Self-Organizing Networks (SON); Self-Healing Concepts and Requirements (Release 11)," 2012-09, v11.0.0.

[7] M. Amirijoo, L. Jorguseski, T. Kurner, R. Litjens, M. Neuland, L. Schmelz, and U. Turke, "Cell Outage Management in LTE Networks," in ISWCS, Sept. 2009, pp. $600-604$

[8] M. Amirijoo, L. Jorguseski, R. Litjens, and L.-C. Schmelz, "Cell Outage Compensation in LTE Networks: Algorithms and Performance Assessment," in IEEE VTC Spring, 2011, pp. 1-5.

[9] M. Amirijoo, L. Jorguseski, R. Litjens, and R. Nascimento, "Effectiveness of Cell Outage Compensation in LTE Networks," in IEEE CCNC, 2011, pp. 642-647.

[10] W. Li, P. Yu, Z. Jiang, and Z. Li, "Centralized Management Mechanism for Cell Outage Compensation in LTE Networks," International Journal of Distributed Sensor Networks, vol. 2012, 2012.

[11] L. Fuqiang, Q. Xuesong, W. Honglin, T. Zhengxian, and M. Luoming, "An Algorithm Of Cell Outage Compensation In Wireless Access Networks," IJACT, vol. 4, no. 1, pp. 404-414, Jan. 2012.

[12] A. Zoha, A. Saeed, A. Imran, M. A. Imran, and A. Abu-Dayya, "A SON Solution for Sleeping Cell Detection using Low-Dimensional Embedding of MDT Measurements," in IEEE PIMRC, Sep. 2014.

[13] Q. Liao, M. Wiczanowski, and S. Stanczak, "Toward Cell Outage Detection with Composite Hypothesis Testing," in IEEE ICC, June 2012, pp. 4883-4887.

[14] R. Barco, V. Wille, and L. Díez, "System for Automated Diagnosis in Cellular Networks Based on Performance Indicators," European Transactions on Telecommunications, vol. 16, no. 5, pp. 399-409, 2005.

[15] B. Cheung, S. G. Fishkin, G. N. Kumar, and S. A. Rao, "Method of Monitoring Wireless Network Performance," US Patent 10/946,255, Sep. 21, 2004.

[16] Y. Ma, M. Peng, W. Xue, and X. Ji, "A Dynamic Affinity Propagation Clustering Algorithm for Cell Outage Detection in Self-Healing Networks," in IEEE WCNC, Apr. 2013, pp. 2266-2270.

[17] A. Coluccia, A. D'Alconzo, and F. Ricciato, "Distribution-Based Anomaly Detection via Generalized Likelihood Ratio Test: A General
Maximum Entropy Approach," Computer Networks, vol. 57, no. 17, pp. 3446-3462, 2013.

[18] R. Khanafer, B. Solana, J. Triola, R. Barco, L. Moltsen, Z. Altman, and P. Lázaro, "Automated Diagnosis for UMTS Networks Using Bayesian Network Approach,” IEEE Trans. Veh. Technol., vol. 57, no. 4, pp. 24512461, 2008.

[19] D. Lopez-Perez, I. Guvenc, G. de la Roche, M. Kountouris, T. Quek, and J. Zhang, "Enhanced Intercell Interference Coordination Challenges in Heterogeneous Networks," IEEE Wireless Commun. Mag., vol. 18, no. 3, pp. 22-30, Jun. 2011.

[20] S. Liu, J. Wu, C. H. Koh, and V. K. N. Lau, "A $25 \mathrm{~Gb} / \mathrm{s}\left(/ \mathrm{km}^{2}\right) \mathrm{Ur}-$ ban Wireless Network Beyond IMT-Advanced," IEEE Commun. Mag., vol. 49, no. 2, pp. 122-129, 2011.

[21] A. Capone, I. Filippini, B. Gloss, and U. Barth, "Rethinking Cellular System Architecture for Breaking Current Energy Efficiency Limits," in Sustainable Internet and ICT for Sustainability (SustainIT), 2012, pp. $1-5$.

[22] H. Ishii, Y. Kishiyama, and H. Takahashi, "A Novel Architecture for LTE-B :C-plane/U-plane Split and Phantom Cell concept," in IEEE Globecom Workshops (GC Wkshps), Dec. 2012, pp. 624-630.

[23] X. Xu, G. He, S. Zhang, Y. Chen, and S. Xu, "On Functionality Separation for Green Mobile Networks: Concept Study over LTE," IEEE Commun. Mag., vol. 51, no. 5, pp. 82-90, May 2013.

[24] A. Zakrzewska, D. López-Pérez, S. Kucera, and H. Claussen, "Dual Connectivity in LTE HetNets with Split Control- and User-Plane," in IEEE Globecom Workshops (GC Wkshps), Dec. 2013, pp. 391-396.

[25] A. Mohamed, O. Onireti, Y. Qi, A. Imran, M. Imran, and R. Tafazolli, "Physical Layer Frame in Signalling-Data Separation Architecture: Overhead and Performance Evaluation," in European Wireless, May 2014.

[26] A. Mohamed, O. Onireti, M. A. Imran, and R. Tafazolli, "Correlationbased Adaptive Pilot Pattern in Control/Data Separation Architecture," in to appear in IEEE ICC, 2015.

[27] T. Alsedairy, Y. Qi, A. Imran, M. A. Imran, and B. Evans, "Self Organizing Cloud cells: A Resource Efficient Network Densification Strategy," Accepted in Transactions on Emerging Telecommunication Technologies.

[28] W. Xue, M. Peng, Y. Ma, and H. Zhang, "Classification-based Approach for Cell Outage Detection in Self-healing Heterogeneous Networks," in IEEE Wireless Communications and Networking Conference (WCNC), Apr. 2014, pp. 2822-2826.

[29] W. Xue, H. Zhang, Y. Li, D. Liang, and M. Peng, "Cell Outage Detection and Compensation in Two-Tier Heterogeneous Networks," International Journal of Antennas and Propagation, 2014.

[30] K. Lee, H. Lee, and D.-H. Cho, "Collaborative Resource Allocation for Self-Healing in Self-Organizing Networks," in IEEE ICC, 2011, pp. 1-5.

[31] 3GPP TS 37.320, "Universal Mobile Telecommunications System (UMTS); LTE; Universal Terrestrial Radio Access (UTRA) and Evolved Universal Terrestrial Radio Access (E-UTRA); Radio measurement collection for Minimization of Drive Tests (MDT); Overall description; Stage 2," 2011-04, v10.1.0 Release 10.

[32] S. Hämälïnen, H. Sanneck, and C. Sartori, LTE Self-Organising Networks (SON): Network Management Automation for Operational Efficiency. John Wiley \& Sons, 2012. 
[33] T. Cox and M. A. Cox, Multidimesional Scaling. CRC Press, 2010.

[34] P. Pawliczek and W. Dzwinel, "Interactive Data Mining by Using Multidimensional Scaling," Procedia Computer Science, vol. 18, no. 0, pp. 40 - 49, 2013, international Conference on Computational Science.

[35] H. Bunke, P. Dickinson, A. Humm, C. Irniger, and M. Kraetzl, "Computer Network Monitoring and Bbnormal Event Detection Using Graph Matching and Multidimensional Scaling," in Advances in Data Mining. Applications in Medicine, Web Mining, Marketing, Image and Signal Mining. Springer, 2006, pp. 576-590.

[36] R. Kharal, "Semidefinite Embedding for the Dimensionality Reduction of DNA Microarray Data," 2006.

[37] M. Zhao and V. Saligrama, "Outlier Detection via Localized p-value Estimation," in Annual Allerton Conference on Communication, Control, and Computing, 2009, pp. 1482-1489.

[38] M. M. Breunig, H.-P. Kriegel, R. T. Ng, and J. Sander, "LOF: Identifying Density-Based Local Outliers," in SIGMOD Conference, 2000, pp. 93 104.

[39] B. L. Deng, "Introduction to Grey System," The Journal of Grey System, vol. 1, pp. 1-24, 1989.

[40] S.-T. Sheu and C.-C. Wu, "Using Grey Prediction Theory to Reduce Handoff Overhead in Cellular Communication Systems," in IEEE PIMRC, vol. 2, 2000, pp. 782-786.

[41] C.-H. Lee and C.-J. Yu, "An Intelligent Handoff Algorithm for Wireless Communication Systems Using Grey Prediction and Fuzzy Decision System," in IEEE ICNSC, 2004, pp. 541-546.

[42] E. Kayacan, B. Ulutas, and O. Kaynak, "Grey System Theory-Based Models in Time Series Prediction," Expert Syst. Appl., vol. 37, no. 2, pp. 1784-1789, 2010.

[43] R. Luo, O. Chen, and S. Pan, "Mobile User Localization in Wireless Sensor Network Using Grey Prediction Method," in 31st Annual Conference of IEEE Industrial Electronics Society, 2005.

[44] L. Panait and S. Luke, "Cooperative Multi-Agent Learning: The State of the Art," Autonomous Agents and Multi-Agent Systems, vol. 3, pp. $383-434,2005$.

[45] R. S. Sutton and A. G. Barto, Reinforcement Learning: An Introduction. MIT Press, Cambridge, 1998.

[46] P. Giuseppe, N. Baldo, and M. Miozzo, "An LTE Module for the ns-3 Network Simulator," in Wns3 (in conjunction with SimuTOOLS), Barcelona, Spain 2011.

[47] 3GPP R4-092042, "Simulation Assumptions and Parameters for FDD HeNB RF Requirements," 2009.

[48] A. P. Bradley, "The use of the Area under the ROC Curve in the Evaluation of Machine Learning Algorithms," Pattern Recognition, vol. 30, no. 7, pp. 1145-1159, 1997.

[49] A. Saeed, O. Aliu, and M. Imran, "Controlling Self Healing Cellular Networks using Fuzzy Logic," in IEEE Wireless Communications and Networking Conference (WCNC), April, Paris, France 2012.

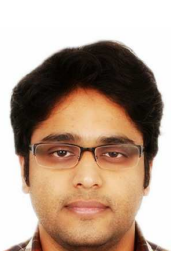

Dr. Ahmed Zoha has completed his Engineering and Physical Sciences Research Council (EPSRC) funded Ph.D. degree in Electronic Engineering from University of Surrey, UK, in 2014. Ahmed is currently working as a Research Scientist in Qatar Mobility Innovation Center (QMIC), Doha, focusing on Self-Organizing Network solutions, particularly automated fault detection and self-healing aspects of cellular network. His research interests include machine learning, big data analytics, knowledge discovery, and self-organizing networks.

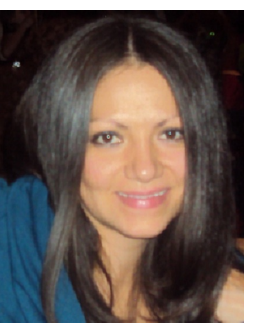

Jessica Moysen holds a BSc in Electronics and Communications Engineering from Instituto Tecnológico y de Estudios Superiores de Monterrey Campus Estado de México (ITESM-CEM), México. She received her MSc degree in Multimedia and Communications from Universidad Carlos III de Madrid, Spain in 2011. In February 2012 she joined CTTC with a grant of the Spanish Ministry of Science and Innovations to pursue a $\mathrm{PhD}$ degree in Telecommunication Engineering at the Signal Theory and Communication department (TSC) of the Universitat Politécnica de Catalunya (UPC). Her research interests include self-organizing networks, wireless networks, machine learning, and statistical tools to analyse data.

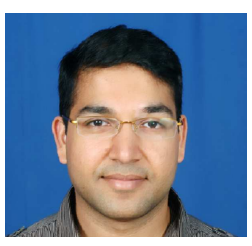

Ali Imran is an assistant professor in telecommunications at University of Oklahoma. $\mathrm{He}$ is currently leading a multinational $\$ 1.045$ million research project on Self Organizing Cellular Networks, QSON (www.qson.org). His research interest include, self-organizing networks, radio resource management and big data analytics. He has authored over 40 peer reviewed articles and has presented number of tutorials at international forums such as IEEE ICC, IEEE WCNC, European Wireless and CrownCom on these topics. He is an Associate Fellow of Higher Education Academy (AFHEA), UK and Member of Advisory Board to Special Technical Community on Big Data at IEEE Computer Society.

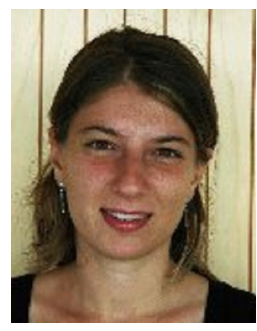

Lorenza Giupponi received the Telecommunications Engineering degree at University of Rome La Sapienza in July 2002 and the $\mathrm{PhD}$ at the Dept. of Signal Theory and Communications (TSC) of the Technical University of Catalonia (UPC) in 2007. She joined the Radio Communications Group of UPC in 2003 with a grant of the Spanish Ministry of Education. During 2006 and 2007 she was assistant professor at UPC. In September 2007 she joined the CTTC. Currently she is a Senior Researcher in the Communication Networks Division, in the Mobile Networks Department, and she act as Director of Institutional Relations as a member of CTTC Executive Committee. She has participated in multiple European Research projects, and she has been the principal investigator of national and international research projects. She is an IEEE Senior member. 
Dr Muhammad Ali Imran is currently a Reader (Associate Professor) in the Institute for Communi-

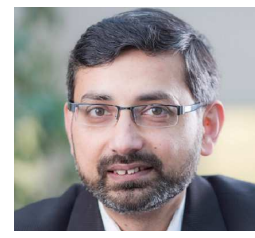
cation Systems (ICS - formerly known as CCSR) at the University of Surrey, UK. He has led a number of multimillion international research projects encompassing the areas of energy efficiency, fundamental performance limits, sensor networks and self-organising cellular networks. He is currently leading the new physical layer work-area for $5 \mathrm{G}$ innovation centre and the curriculum design for the Engineering for Health program at Surrey. He has a global collaborative research network spanning both academia and key industrial players in the field of wireless communications. He has supervised 20 successful $\mathrm{PhD}$ graduates and published over 200 peer-reviewed research papers including more than 20 IEEE Transaction papers. He has delivered several keynotes, plenary talks, invited lectures and tutorials in many international conferences and seminars. He has been a guest editor for special issues in IEEE Communications, IEEE Wireless Communication Magazine, IET Communications and IEEE Access. He is an associate Editor for IEEE Communications Letters and IET Communications Journal. He has been awarded IEEE Comsocs Fred Ellersick award 2014 and FEPS Learning and Teaching award 2014 and twice nominated for Tony Jeans Inspirational Teaching award. He was a shortlisted finalist for The Wharton-QS Stars Awards 2014 for innovative teaching and VCs learning and teaching award in University of Surrey. He is a senior member of IEEE and a Senior Fellow of Higher Education Academy (HEA), UK.

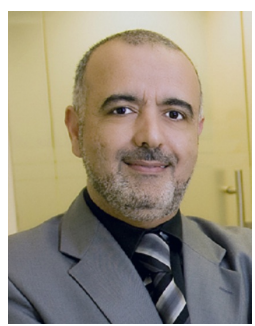

Dr. Adnan Abu-Dayya received his PhD in Electrical Engineering from Queens University, Canada in 1992. He has more than 25 years of global experience in the areas of ICT R\&D, product innovations, business development, and services delivery. Dr. Abu-Dayya is currently appointed as the Founding Executive Director (CEO) of the Qatar Mobility Innovations Center (QMIC) at the Qatar Science \& Technology Park, Qatar. Dr. Abu-Dayya also serves on the advisory boards of the Arab Innovation Network, and the Department of Electrical and Computer Engineer at Texas A\&M University at Qatar. 